\title{
Membrane Theory Treatment of Eccentric Flows in Concentric Hoppers
}

\author{
A.J. Sadowski and J.M. Rotter \\ The University of Edinburgh, Scotland, UK
}

\begin{abstract}
This paper presents an initial study of the effects of an accidental eccentric flow channel that sometimes develops in the conical hopper of a metal storage silo. A simple assumed pressure regime is adopted, based on studies of eccentric discharge in cylindrical silos, and the structural actions are analysed using shell membrane theory. The results are verified against a finite element analysis. A set of equations is derived which give the complete membrane stress state in the hopper under such an unsymmetrical pressure regime.
\end{abstract}

Under symmetrical loading, silo hoppers are subject to biaxial tension, with failure normally governed by material rupture due to tensile meridional membrane stresses. It is found that the eccentric flow channel leads to dramatically increased meridional membrane stresses at the transition. Compressive membrane stresses are also found near the hopper outlet and at the sides of the flow channel near the transition. A reasonably close correlation is found between the derived membrane theory equations in a right circular conical hopper and linear elastic finite element predictions.

Keywords: Thin shell structures, solids flow, structural stability, elastic analysis, material rupture, metal silos, hoppers. 
Published in: Thin-Walled Structures, 49(7), 902-912.

DOI: http://dx.doi.org/10.1016/j.tws.2011.02.016

\section{Introduction}

\subsection{Background}

Silos are important storage structures used extensively in many industries and agriculture to store a wide range of solids. It is acknowledged that the most serious design condition for silos is discharge, and that the unsymmetrical pressures associated with eccentric discharge have been the cause of many silo disasters in the past $[4,11,14,17,18]$. The literature on the behaviour and design of hoppers is very small compared to that of cylindrical silos and has predominantly focused on axisymmetric geometries and mass flow patterns (e.g. [2, 7, 24]). There are a number of reasons for this, as follows.

Firstly, not every silo has a hopper at its base. Secondly, analytical shell bending theory quickly becomes prohibitively difficult for shells other than cylinders, especially under unsymmetrical loads. For example, the bending analysis of a conical shell under axisymmetric loading alone leads to a solution involving Bessel functions, which are not easily applied in design [15]. Using membrane theory, the equilibrium of an unsymmetrically-loaded conical shell leads to a set of partial differential equations with no closed form solution. Cylindrical shells are, in this respect, simpler to solve analytically and standard solutions are widely available in $[8,9,15,16,22,23]$ and others.

Thirdly, it is difficult to characterise pressure distributions in a silo under unsymmetrical flow patterns $[10,13,26]$. For example, the pressures associated with an eccentrically formed parallel-sided channel of discharging solid (see Fig. 1) have been given a realistic codified representation for the first time in the recent European standard EN 1991-4 [6], based on the earlier work of Rotter [14, 18]. Similar but less successful treatments were presented in [10, 21, 26]. For hoppers, however, only axisymmetric pressure distributions (attributed to Walker [25]) have been defined in EN 1991-4 [6], and for load cases other than this, a finite element analysis is recommended.

Lastly, although the analysis of conical shells is mathematically more difficult than cylindrical shells, the structural consequences are less severe under axisymmetric loading. Where the cylindrical sections of the silo are subject to extensive compressive stresses and a buckling limit state, hoppers under symmetrical loads are in a state of biaxial tension and their strength is governed by rupture at the transition. The latter is a simpler design condition and, under 
Published in: Thin-Walled Structures, 49(7), 902-912.

DOI: http://dx.doi.org/10.1016/j.tws.2011.02.016

axisymmetric loads, the membrane forces may be found easily from local static equilibrium with no need for a shell bending theory analysis.

By contrast, unsymmetrical pressures associated with eccentric discharge in hoppers may lead to severe distortion and damage to the transition and ringbeam [17], and the authors are aware of no previous research on the topic. The analytical solution for unsymmetrical pressures developed in this paper may be useful for hopper design and may be used in place of a much more onerous finite element analysis. This paper thus uses membrane theory as the principal analytical vehicle because it gives a clearer insight into the structural behaviour as well as a general solution to the problem.

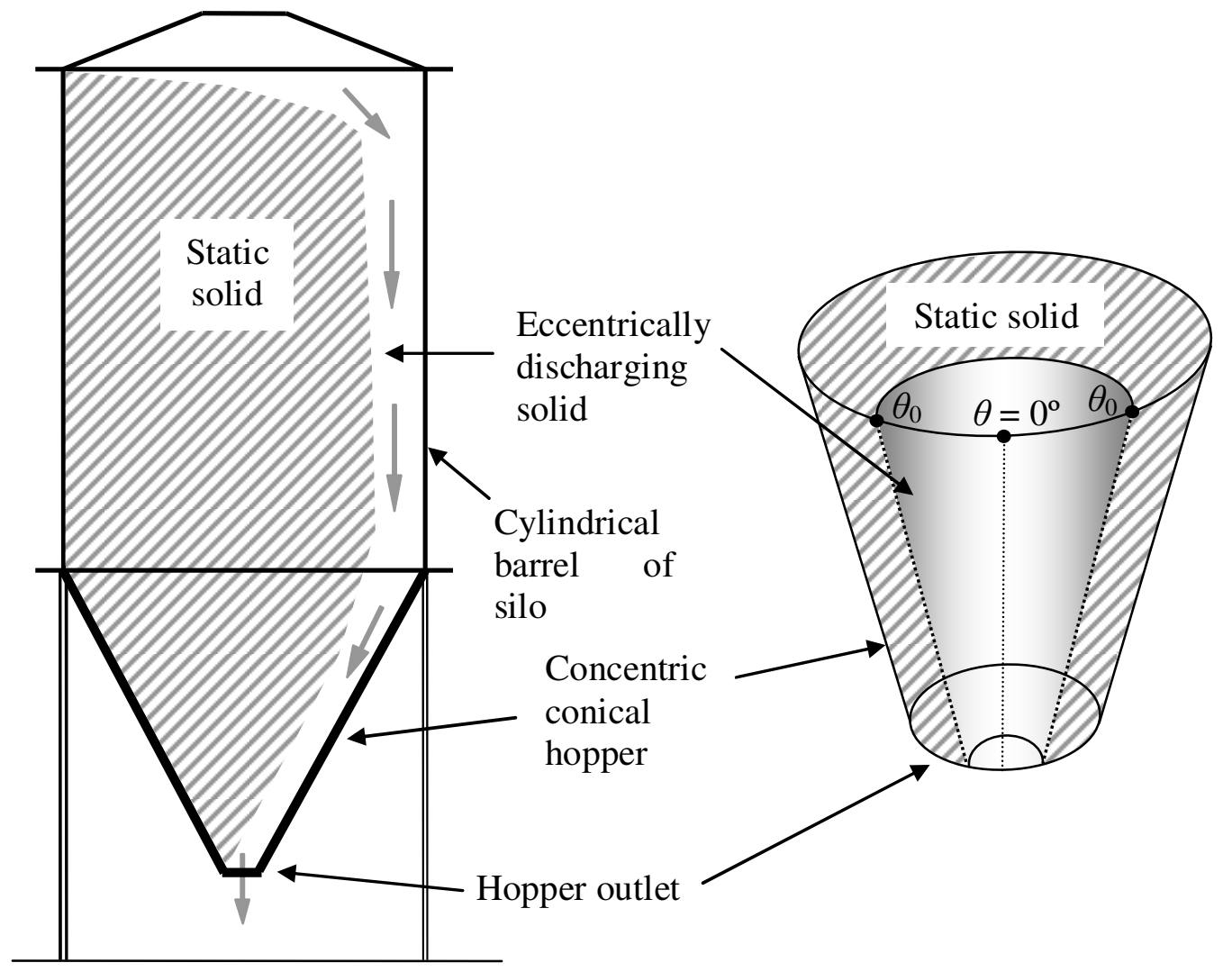

Fig. 1. Select silo terminology and flow pattern during accidental eccentric discharge, after [17]

\subsection{Proposed pressure pattern for eccentric discharge in concentric hoppers}

This paper focuses on a right-circular conical hopper subjected to a pressure distribution illustrative of that occurring in an eccentric channel of discharging solids. It is desirable for a convergent and accurate membrane theory solution that the function of the pressure distribution is continuous up to the second derivative. Before a suitable form can be suggested it is 
Published in: Thin-Walled Structures, 49(7), 902-912.

DOI: http://dx.doi.org/10.1016/j.tws.2011.02.016

necessary to review an existing pressure description for eccentric flow channels in cylindrical silos.

Early research into eccentric discharge treated a cylindrical silo as a simple planar ring [10, 13, 16] subject to circumferential bending moments, which carried the implicit assumption that the silo was constructed in reinforced concrete and would fail by material yielding or fracture. The first work to offer a more holistic three-dimensional treatment of theoretical silo wall pressures under eccentric discharge is the work of Rotter [14]. His pressure model, based on sound considerations of equilibrium, relates to a parallel-sided flow channel forming such that its boundaries touch the silo wall (similar to Fig. 1), is shown in two versions in Fig. 2. The first version shows a depression in normal pressures due to the channel reflecting what has been observed in experiments, the references of which may be found in the above paper. The alternative version contains additional zones of high pressures at the boundaries between the static and flowing solid, as suggested by Wood [26] and found as a small feature in the full scale experiments of Chen et al. [5].

The alternative version is more severe on shell structures and has been incorporated into EN 1991-4 [6] to give a conservative design for large silos or those where high eccentricity of flows are expected. However, high edge pressures represent a dramatic discontinuity and lead to very large curvatures and changes of geometry in the shell, and thus high bending moments, making the application of membrane theory unsuitable. These pressure increases, which are considerably exaggerated in the EN 1991-4 model, were therefore omitted in this study. It has additionally been observed that a local pressure decrease, not an increase, has the greatest deleterious effect on silo stability $[14,16,19,20]$, so it is thought that this assumption is justified at this point. 
Published in: Thin-Walled Structures, 49(7), 902-912.

DOI: http://dx.doi.org/10.1016/j.tws.2011.02.016

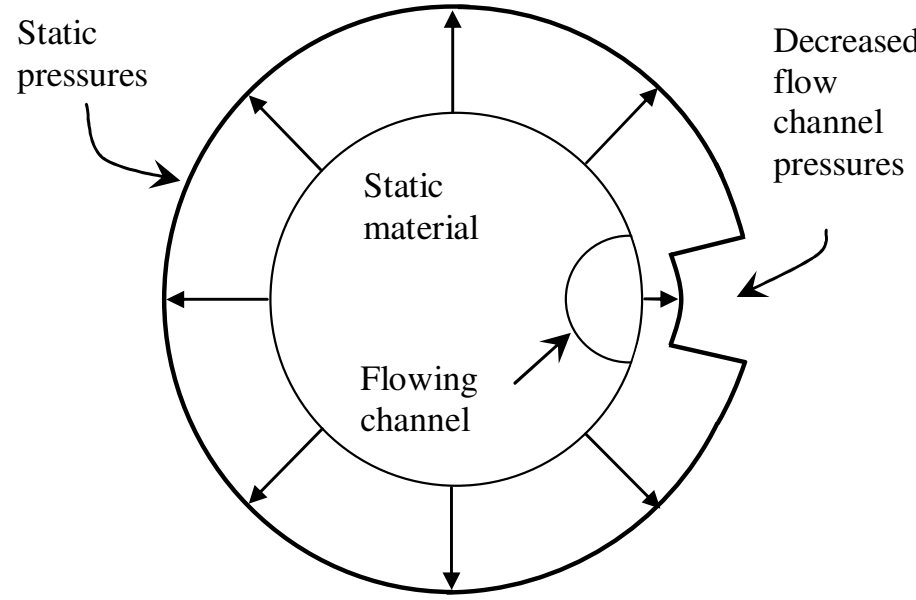

a) without pressure peaks

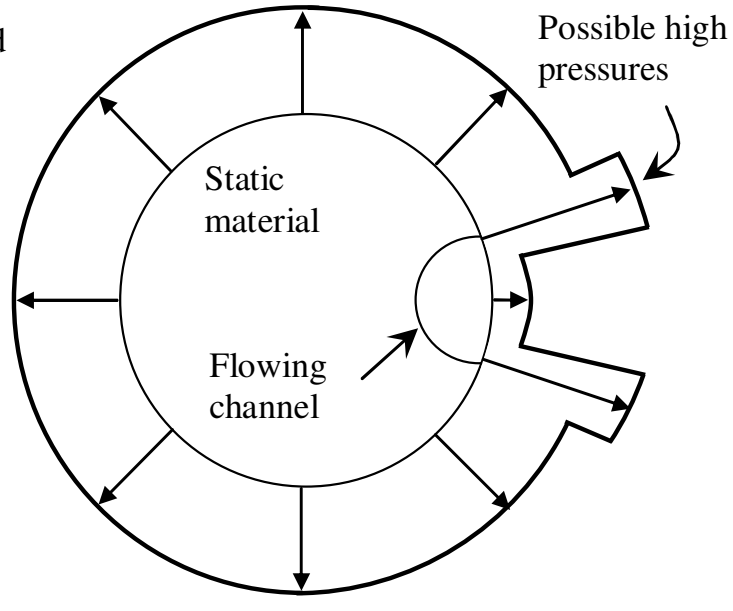

b) with pressure peaks

Fig. 2. Normal pressure distributions under eccentric discharge flows

The above treatment may be extended to hoppers. It is assumed here that the parallel-sided flow channel begins at the outlet, passes up the side of the hopper and continues into the cylinder where it is normally assumed to lie (Fig. 1). The shape of the depression in pressure must be chosen with great care to ensure convergence in the membrane theory analysis, and four different shapes are investigated. Rotter [16] suggested 'rectangular', 'triangular' and 'C1 smooth' shapes, and a further, 'C2 smooth', is added here (see Fig. 3 and Table 1). The rectangular shape is discontinuous in value, the triangular shape is discontinuous in slope (C0 continuous) at its edges and centre, the $\mathrm{C} 1$ smooth shape is discontinuous in curvature at its edges only, while the $\mathrm{C} 2$ smooth shape is continuous in both slope and curvature at its edges. It will become evident later that a high degree of continuity is necessary for successful membrane theory treatments of this kind. 
Published in: Thin-Walled Structures, 49(7), 902-912.

DOI: http://dx.doi.org/10.1016/j.tws.2011.02.016
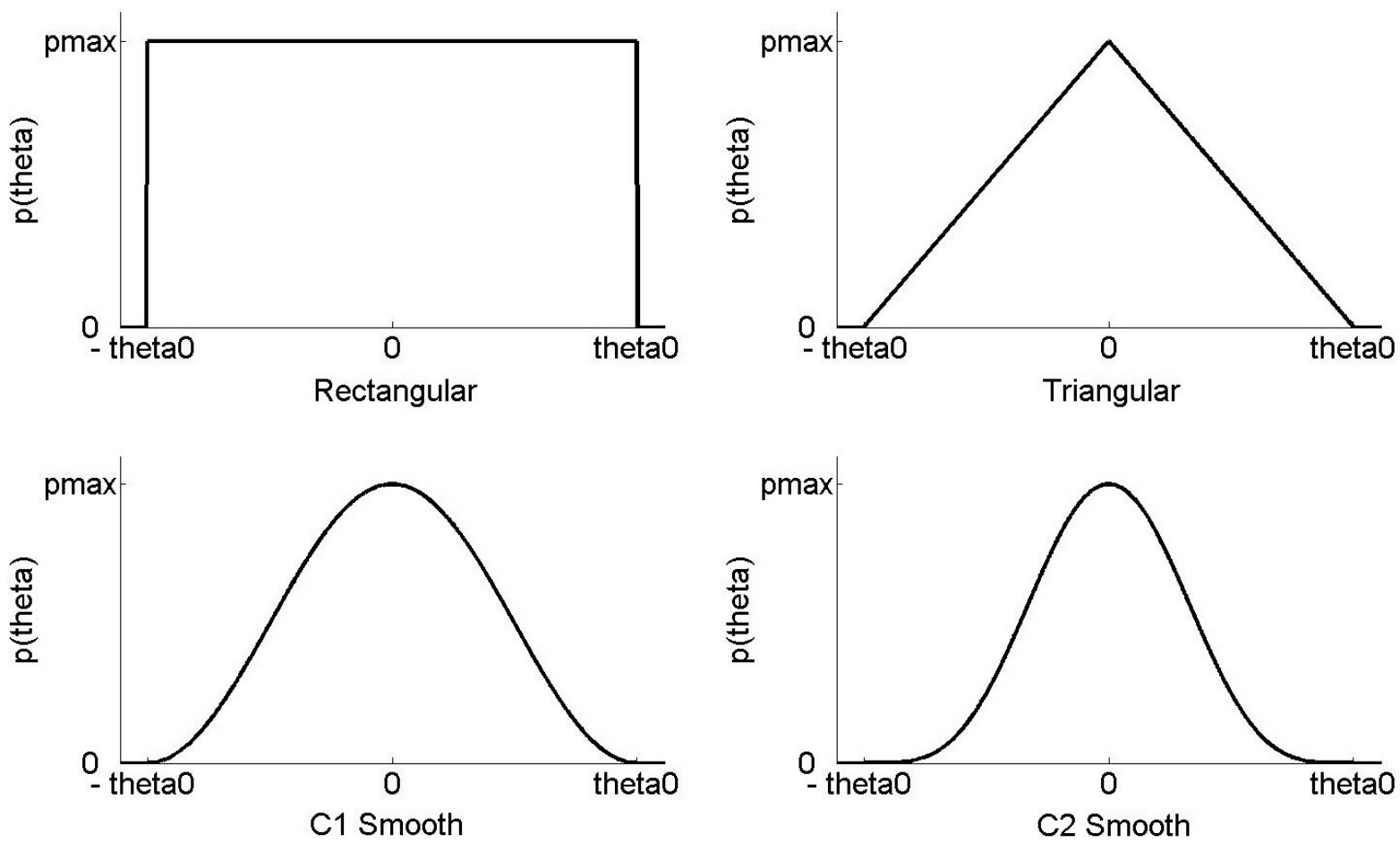

Fig. 3. Suggested shapes of pressure depression due to the flow channel

The depression in pressures is expanded as a Fourier series in the circumferential coordinate with a plane of symmetry at $\theta=0^{\circ}$, so only the cosine terms of the series need to be considered. The amplitude of the depression is arbitrarily chosen as a fraction, $\eta$, of the local values of the normal axisymmetric pressures, $p_{\text {axi }}$ (Eq. 1).

The axisymmetric normal pressures are defined by (EN 1991-4 [6]) as follows:

$p_{a x i}(z)=F p_{v}(z)$

where $p_{v}(z)=\left(p_{v f t}-\frac{\gamma L}{n-1}\right)\left(\frac{z}{L}\right)^{n}+\frac{\gamma L}{n-1}\left(\frac{z}{L}\right)$

$$
F=\frac{1+0.8 \mu \cot \beta}{1+\mu \cot \beta} ; n=2(F \mu \cot \beta+F-1) ; p_{v f t}=\gamma R
$$

These are taken as filling values, with no additional factors.

Two typical shapes of the axisymmetric distribution $p_{\text {axi }}$ are shown in Fig. 4. These change depending on the silo aspect ratio, the choice of the wall pressure ratio $F$ and material properties. 
Published in: Thin-Walled Structures, 49(7), 902-912.

DOI: http://dx.doi.org/10.1016/j.tws.2011.02.016

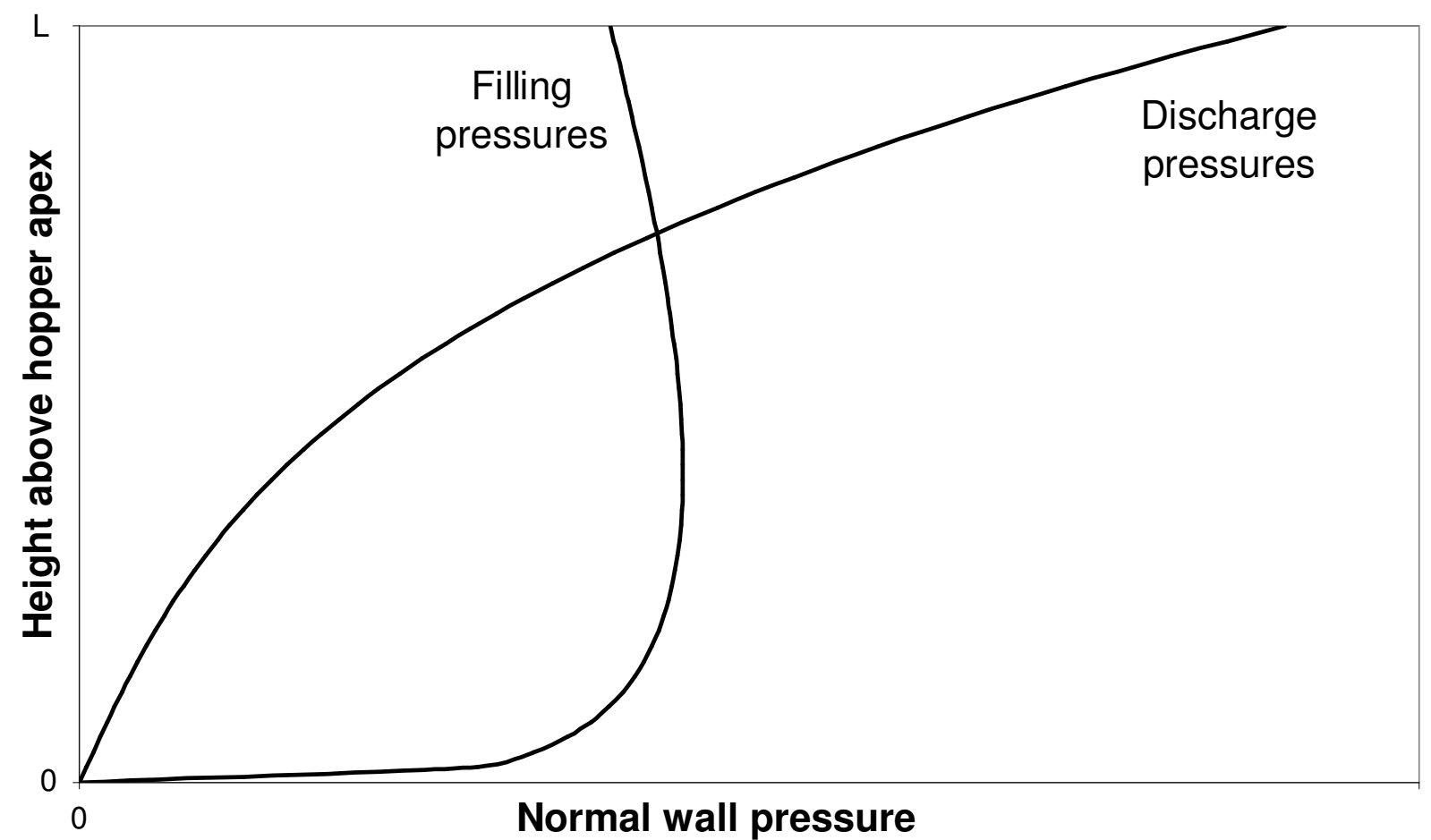

Fig. 4. Example distribution of normal wall pressures in a conical hopper under filling and discharge conditions

The normal surface pressures including the flow channel are then defined by:

$p_{n}(z, \theta)=p_{a x i}(z)+\sum_{r=0}^{\infty} p_{n r}(z) \cos r \theta$

The meridional shear tractions are defined everywhere as:

$p_{\phi}(z, \theta)=-\mu p_{n}(z, \theta)$

The harmonics of the series are given by:

$p_{n r}(z)=f_{r} p_{p}(z)$

where $p_{p}(z)=\eta p_{a x i}(z)$ and $0 \leq r \leq \infty$

The Fourier coefficients $f_{\mathrm{r}}$ depend on the shape of the depression and are given by:

$f_{r}=\left\{\begin{array}{l}\frac{1}{\pi} \int_{0}^{\theta_{0}} p(\theta) d \theta \text { for } r=0 \\ \frac{2}{\pi} \int_{0}^{\theta_{0}} p(\theta) \cos r \theta d \theta \text { for } r>0\end{array}\right.$

For higher harmonics, Gibb's phenomenon may occur which is eliminated by sigma smoothing [12]:

$f_{r, \text { smooth }}=\sigma_{r} f_{r}$ 
Published in: Thin-Walled Structures, 49(7), 902-912.

DOI: http://dx.doi.org/10.1016/j.tws.2011.02.016

where $\sigma_{r}=\operatorname{sinc}\left(\frac{r \pi}{r_{\max }}\right)=\sin \left(\frac{r \pi}{r_{\max }}\right)\left(\frac{r_{\max }}{r \pi}\right)$

Table 1. Fourier series expansions for the depression shapes

\begin{tabular}{|l|c|c|c|}
\hline Name & $\begin{array}{c}\text { Shape of } p(\theta) \text { and } \\
\text { range }\end{array}$ & $\begin{array}{c}\text { Coefficient } \\
f_{0}, r=0\end{array}$ & $\begin{array}{c}\text { Coefficient } \\
f_{\mathrm{r}}, r>0\end{array}$ \\
\hline Rectangular & $p_{\max }$ & $\frac{2 \sin \left(r \theta_{0}\right)}{\pi r}$ & $\frac{\theta_{0}}{\pi}$ \\
\hline Triangular & $\left(1-\frac{\theta}{\theta_{0}}\right) p_{\max }$ & $\frac{\theta_{0}}{2 \pi}$ & $\frac{2\left(1-\cos \left(r \theta_{0}\right)\right)}{\pi r^{2} \theta_{0}}$ \\
\hline C1 Smooth & $\frac{1}{2}\left(1+\cos \left(\frac{\pi \theta}{\theta_{0}}\right)\right) p_{\max }$ & $\frac{\theta_{0}}{2 \pi}$ & $\frac{\pi \sin \left(r \theta_{0}\right)}{r\left(\pi^{2}-r^{2} \theta_{0}^{2}\right)}$ for $r \neq \frac{\pi}{\theta_{0}}$ \\
& $-\theta_{0} \leq \theta \leq \theta_{0}$ & $\frac{\theta_{0}}{2 \pi}$ for $r=\frac{\pi}{\theta_{0}}$ \\
\hline C2 Smooth & $\cos ^{4}\left(\frac{\pi \theta}{2 \theta_{0}}\right) p_{\max }$ & $\frac{3 \theta_{0}}{8 \pi}$ & $\frac{3 \pi^{3} \sin \left(r \theta_{0}\right)}{r\left(r^{4} \theta_{0}^{4}-5 r^{2} \theta_{0}^{2} \pi^{2}+4 \pi^{4}\right)}$ for $r \neq \frac{\pi}{\theta_{0}}, \frac{2 \pi}{\theta_{0}}$ \\
\hline
\end{tabular}

\section{Shell membrane theory for conical shells and derivation of general expressions}

\subsection{Introduction and derivation of the governing differential equations}

The rupture mechanism through which the hopper is likely to fail during eccentric discharge results in little bending in the meridional direction, hence it is appropriate to analyse the hopper using shell membrane theory. Membrane theory may be applied to the analysis of shells provided that the loads vary smoothly with no discontinuities. Hence much care is required in choosing an appropriate function for the pressure depression. The membrane stress state in the hopper may be calculated relatively accurately, except near the transition and near the base where bending occurs to maintain compatibility with restrained boundary displacements. These regions of bending are expected to be limited to within about two meridional bending half-wavelengths of either boundary, closely approximated by [15]: 
Published in: Thin-Walled Structures, 49(7), 902-912.

DOI: http://dx.doi.org/10.1016/j.tws.2011.02.016

$$
\lambda=\frac{\pi}{\left[3(1-v)^{2}\right]^{0.25}}\left(\frac{R t}{\cos \beta}\right)^{0.5}
$$

Unlike cylinders where $\beta=0$ and $\lambda$ takes its minimum value, the region of bending extends further into a conical shell when $\beta>0$. The following analysis is therefore relevant to moderately steep hoppers, with smoothly varying pressure patterns [16].

If the bending and twisting moments and transverse shear forces are ignored, there remain three membrane stress resultants in the shell induced by distributed normal pressures and both meridional and circumferential frictional tractions. An infinitesimally small segment of the wall of a conical shell is shown in Fig. 5. The membrane stress resultants are constant through the shell thickness and are defined as:

$$
N_{\theta}=t \sigma_{\theta} ; N_{\phi \theta}=t \tau_{\phi \theta} ; N_{\phi}=t \sigma_{\phi}
$$

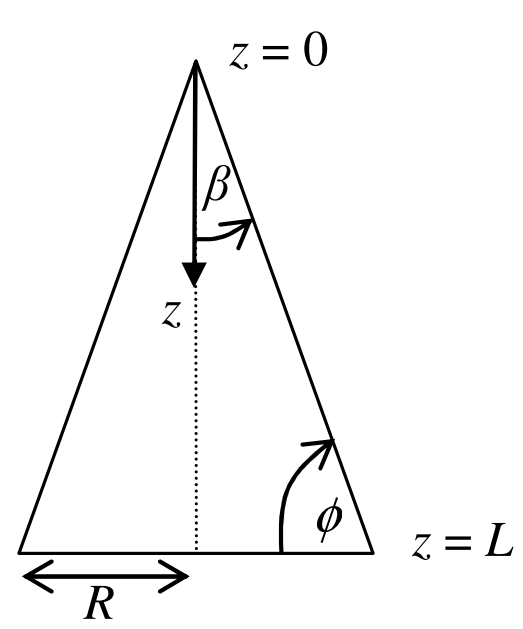

a) Cone/hopper geometry

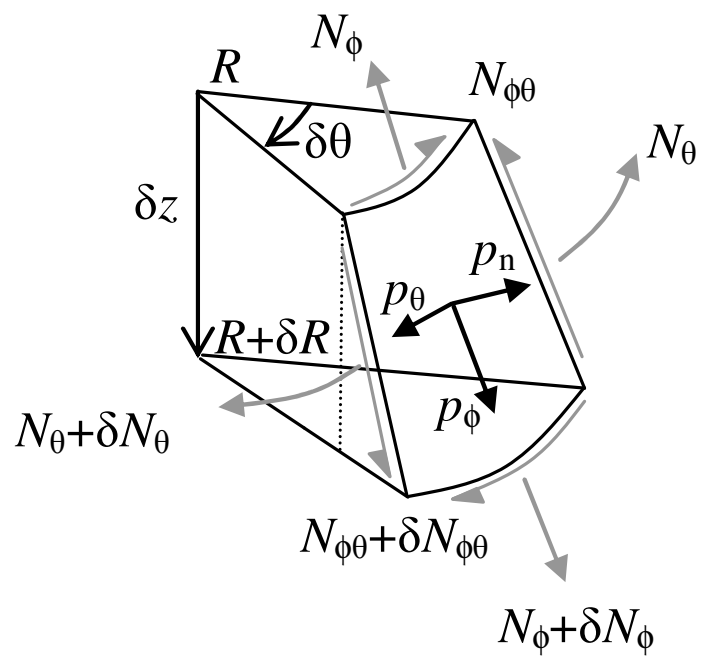

b) Membrane stress resultants in a shell element

Fig. 5. Hopper geometry and static equilibrium of membrane forces

The three equations derived from static equilibrium of a shell element are as follows:

Normal: $N_{\theta}=p_{n} z \sec \beta \tan \beta$

Circumferential: $2 N_{\phi \theta}+z \frac{\partial N_{\phi \theta}}{\partial z}=-z \sec \beta\left(p_{\theta}+\sec \beta \frac{\partial p_{n}}{\partial \theta}\right)$

Meridional: $N_{\phi}+z \frac{\partial N_{\phi}}{\partial z}=-\csc \beta \frac{\partial N_{\phi \theta}}{\partial \theta}+z \sec \beta\left(p_{n} \tan \beta-p_{\phi}\right)$

It is necessary to simplify these equations before they can be solved. The method used here is discussed in the next sections. It should be noted that the PDE governing membrane shear (Eq. 
Published in: Thin-Walled Structures, 49(7), 902-912.

DOI: http://dx.doi.org/10.1016/j.tws.2011.02.016

10) involves the circumferential derivative of the normal pressure $p_{\mathrm{n}}$, so the rectangular pressure distribution of Fig. 3 is immediately unusable. The PDE governing the meridional membrane stress resultant $N_{\phi}$ involves the second derivative of $p_{\mathrm{n}}$, so even the triangular distribution, which is discontinuous in its first derivative, is also unusable. The consequences of these discontinuities are seen later.

\subsection{Membrane stress resultants for general axisymmetric pressures}

For axisymmetric pressures there is no variation with respect to the circumferential coordinate. All derivatives with respect to $\theta$ therefore vanish, as do the circumferential tractions $p_{\theta}$ and the membrane shear stress resultant $N_{\phi \theta}$. The circumferential equilibrium equation (Eq. 10) vanishes, and the meridional equilibrium equation (Eq. 11) reduces to an ordinary differential equation with a simple solution:

$N_{\phi}+z \frac{d N_{\phi}}{d z}=z \sec \beta\left(p_{n} \tan \beta-p_{\phi}\right)$

The solution for the three stress resultants is therefore:

$$
\begin{aligned}
& N_{\theta}(z)=p_{n}(z) z \sec \beta \tan \beta \\
& N_{\phi \theta}(z)=0 \\
& N_{\phi}(z)=\frac{1}{z} \int_{0}^{z} g(z) d z+N_{\phi 0} \text { where } g(z)=z \sec \beta\left(p_{n}(z) \tan \beta-p_{\phi}(z)\right)
\end{aligned}
$$

The constant of integration $\left(N_{\phi 0}\right)$ in Eq. 15 is zero corresponding to a physical boundary condition at a stress-free bottom outlet.

\subsection{Membrane stress resultants for general non-symmetric pressures}

For non-symmetric pressures, the membrane stress resultants may be expressed as a multiple Fourier series in $\theta$ :

$$
\begin{aligned}
& N_{\theta}(z, \theta)=\sum_{r=0}^{\infty} N_{\theta r}(z) \cos r \theta \\
& N_{\phi \theta}(z, \theta)=\sum_{r=0}^{\infty} N_{\phi \theta r}(z) \sin r \theta \\
& N_{\phi}(z, \theta)=\sum_{r=0}^{\infty} N_{\phi r}(z) \cos r \theta
\end{aligned}
$$


Published in: Thin-Walled Structures, 49(7), 902-912.

DOI: http://dx.doi.org/10.1016/j.tws.2011.02.016

The Fourier coefficients are given in Table 1 depending on the assumed depression form of the pressure depression. Substituting Eqs 16 to 18 into Eqs 9 to 11 allows a simplified set of equilibrium equations to be derived for each harmonic:

Normal: $N_{\theta r}=p_{n r} z \sec \beta \tan \beta$

Circumferential: $2 N_{\phi \theta r}+z \frac{d N_{\phi \theta r}}{d z}=-z \sec \beta\left(p_{\theta r}-r \sec \beta p_{n r}\right)$

Meridional: $N_{\phi r}+z \frac{d N_{\phi r}}{d z}=-r N_{\phi \theta r} \csc \beta+z \sec \beta\left(p_{n r} \tan \beta-p_{\phi r}\right)$

These are now ordinary differential equations which may be solved per harmonic:

$N_{\theta r}(z)=p_{n r}(z) z \sec \beta \tan \beta$

$N_{\phi \theta r}(z)=-\frac{1}{z^{2}} \int_{0}^{z} g(z) d z+N_{\phi \theta r 0}$ where $g(z)=z^{2} \sec \beta\left(p_{\theta r}(z)-r \sec \beta p_{n r}(z)\right)$

$N_{\phi r}(z)=\frac{1}{z} \int_{0}^{z} h(z) d z+N_{\phi r 0}$ where $h(z)=-r N_{\phi \theta r}(z) \csc \beta+z \sec \beta\left(p_{n r}(z) \tan \beta-p_{\phi r}(z)\right)$

The two constants of integration, $N_{\phi \theta \mathrm{r} 0}$ and $N_{\phi \mathrm{r} 0}$, require special attention because the bottom edge of a conical hopper cannot be completely free under unsymmetrical loading as it will lead to large distortions of geometry and of the membrane stress state. Boundary conditions are not straightforward for cones, but here a state of zero shear $\left(N_{\phi \theta r}=0\right)$ is assumed. The constant of integration for the meridional membrane stress resultant, $N_{\phi \mathrm{r} 0}$, requires special treatment and is described below.

\section{Derivation of membrane stress resultants for specific cases of loading}

It is now possible to describe the full membrane stress state in a hopper subjected to both axisymmetric and harmonically-varying pressures.

\subsection{Axisymmetric pressures}

For a hopper subjected to axisymmetric pressures only as defined by Eq. 1, the membrane stress resultants are the following equations (Rotter, 2001a):

$$
\begin{aligned}
& N_{\theta}(z)=F \sec \beta \tan \beta\left[\left(p_{v f t}-\frac{\gamma L}{n-1}\right)\left(\frac{z^{n+1}}{L^{n}}\right)+\frac{\gamma}{n-1}\left(z^{2}\right)\right] \\
& N_{\phi}(z)=F \sec \beta(\tan \beta+\mu)\left[\left(p_{v f t}-\frac{\gamma L}{n-1}\right)\left(\frac{z^{n+1}}{(n+2) L^{n}}\right)+\frac{\gamma}{n-1}\left(\frac{z^{2}}{3}\right)\right]
\end{aligned}
$$


Published in: Thin-Walled Structures, 49(7), 902-912.

DOI: http://dx.doi.org/10.1016/j.tws.2011.02.016

\subsection{Non-symmetric pressures}

The membrane stress resultants due to the depression of Fig. 3 are given for any harmonic $r$ by:

$$
\begin{aligned}
& N_{\theta r}(z)=\eta F f_{r} \sec \beta \tan \beta\left[\left(p_{v f t}-\frac{\gamma L}{n-1}\right)\left(\frac{z^{n+1}}{L^{n}}\right)+\frac{\gamma}{n-1}\left(z^{2}\right)\right] \\
& N_{\phi \theta r}(z)=\eta F f_{r} r \sec ^{2} \beta\left[\left(p_{v f t}-\frac{\gamma L}{n-1}\right)\left(\frac{z^{n+1}}{(n+3) L^{n}}\right)+\frac{\gamma}{n-1}\left(\frac{z^{2}}{4}\right)\right]-\frac{1}{z^{2}} \sec \beta I_{\theta r}+N_{\phi \theta r 0}
\end{aligned}
$$

where $I_{\theta r}=\int_{0}^{z} z^{2} p_{\theta r} d z$

$$
\begin{aligned}
N_{\phi r}(z)= & \eta F f_{r} \sec \beta\left[\left((\tan \beta+\mu)-\frac{r^{2} \csc \beta \sec \beta}{n+3}\right)\left(p_{v f t}-\frac{\gamma L}{n-1}\right)\left(\frac{z^{n+1}}{(n+2) L^{n}}\right)+\ldots\right. \\
& \left.\ldots\left((\tan \beta+\mu)-\frac{r^{2} \csc \beta \sec \beta}{4}\right)\left(\frac{\gamma}{n-1}\right)\left(\frac{z^{2}}{3}\right)\right]+r \csc \beta\left(\frac{\sec \beta}{z} I I_{\theta r}-N_{\phi \theta r 0}\right)+N_{\phi r 0}
\end{aligned}
$$

where $I I_{\theta r}=\int_{0}^{z} \frac{1}{z^{2}} I_{\theta r} d z=\int_{0}^{z} \frac{1}{z^{2}}\left[\int_{0}^{z} z^{2} p_{\theta r} d z\right] d z$

The expressions $I_{\theta \mathrm{r}}$ and $I I_{\theta \mathrm{r}}$ include the contribution from circumferential frictional tractions, $p_{\theta}$. These have been retained for completeness, but since no data is available on their form, they are set to zero for the numerical studies of this paper. They are probably negligible unless there is a significant circumferential flow in the stored solid in the hopper.

\subsection{Base meridional restraint}

The method of linking membrane theory with strain-displacement and constitutive relations from the theory of elasticity used by Rotter [16] was employed to derive the meridional integration constant per harmonic $N_{\phi r}$. This treatment is necessary to enforce a meridional displacement boundary condition, for which there is no scope in membrane theory which is based equilibrium alone.

The meridional strain-displacement relationship for an axisymmetric conical shell adapted from the relation for curved shells from Sander's shell theory [3] is given by:

$\varepsilon_{\phi}(z)=\cos \beta \frac{d u}{d z}$

The constitutive relation for an isotropic material is also employed (e.g. [8]):

$\varepsilon_{\phi}(z)=\frac{1}{E t}\left(N_{\phi}(z)-v N_{\theta}(z)\right)$ 
Published in: Thin-Walled Structures, 49(7), 902-912.

DOI: http://dx.doi.org/10.1016/j.tws.2011.02.016

Equating and solving for $u$ gives:

$$
u(z)=\frac{\cos \beta}{E t} \int_{0}^{z}\left(N_{\phi}(z)-v N_{\theta}(z)\right) d z+u_{0}
$$

The meridional displacement $u$ may be expanded as a Fourier series in the same manner as the membrane stress resultants (Eqs 16 to 18$)$ :

$$
u(z)=\sum_{r=0}^{\infty} u_{r}(z) \cos r \theta
$$

Implementing Eqs 16 to 18 and Eq. 33 in Eq. 32 gives:

$$
u_{r}(z)=\frac{\cos \beta}{E t}\left[\int_{0}^{z}\left(N_{\phi r \ell}(z)-v N_{\theta r \ell}(z)\right) d z+z\left(N_{\phi r 0}-r \csc \beta N_{\phi \theta r 0}\right)\right]+u_{0 r}
$$

where $N_{\phi r \ell}(z)=N_{\phi r}(z)-N_{\phi r 0}$ and $N_{\theta r \ell}(z)=N_{\theta r}(z)$

Under a meridional displacement restraint at the base and transition, $u_{\mathrm{r}}(0)=u_{\mathrm{r}}(L)=0$ for all $r$, and a shear-free base, $N_{\phi \theta r}=0$ for all $r$, the meridional integration constant per harmonic is obtained:

$$
N_{\phi r 0}=-\eta L F f_{r} \sec \beta\left[\begin{array}{l}
\left(p_{v f t}-\frac{\gamma L}{n-1}\right)\left(\frac{\tan \beta+\mu}{(n+2)^{2}}-\frac{r^{2} \sec \beta \csc \beta}{(n+3)(n+2)^{2}}-\frac{v \tan \beta}{n+2}\right) \cdots \\
+\left(\frac{\gamma L}{n-1}\right)\left(\frac{\tan \beta+\mu}{9}-\frac{r^{2} \sec \beta \csc \beta}{36}-\frac{v \tan \beta}{3}\right)
\end{array}\right]
$$

\subsection{Convergence study}

Following the above solution, the convergence of the membrane stress resultant was investigated for higher harmonics. The convergence is governed by the Fourier coefficient $f_{\mathrm{r}}$, and depends on the function used to describe the pressure depression and the continuity of its first and second derivatives, which appear in the circumferential and meridional equilibrium equations (Eqs 10 and 11) respectively. If the terms diverge at higher harmonics, then the chosen function is unsuitable for this analysis.

The normalised values of the terms of all three membrane stress resultants up to the $40^{\text {th }}$ harmonic are shown in Fig. 6 without sigma smoothing and Fig. 7 with sigma smoothing (Eq. 6) for all four depression functions. The thickest line on each curve represents the meridional membrane stress resultant harmonic, which is the most important stress component from a structural resistance perspective for this load case. It is obtained after double differentiation of the pressure depression function with respect to $\theta$ and is thus also the most problematic in terms 
Published in: Thin-Walled Structures, 49(7), 902-912.

DOI: http://dx.doi.org/10.1016/j.tws.2011.02.016

of convergence. The circumferential and shear membrane stress resultant harmonics are of less importance and converge faster, and they are not separately identified in Figs 6 and 7.

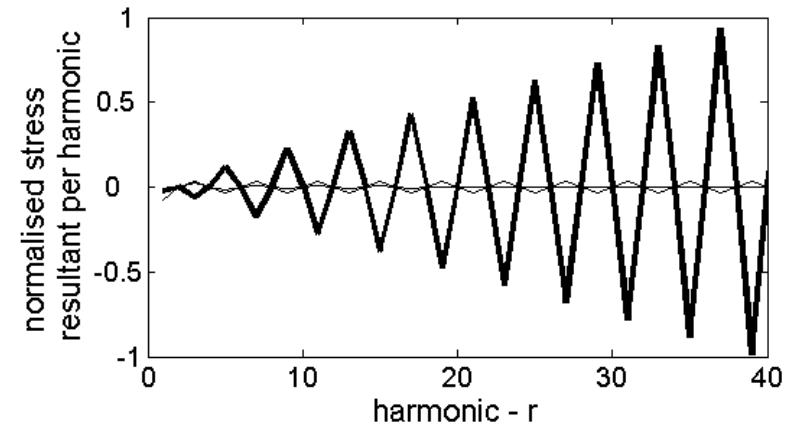

Rectangular

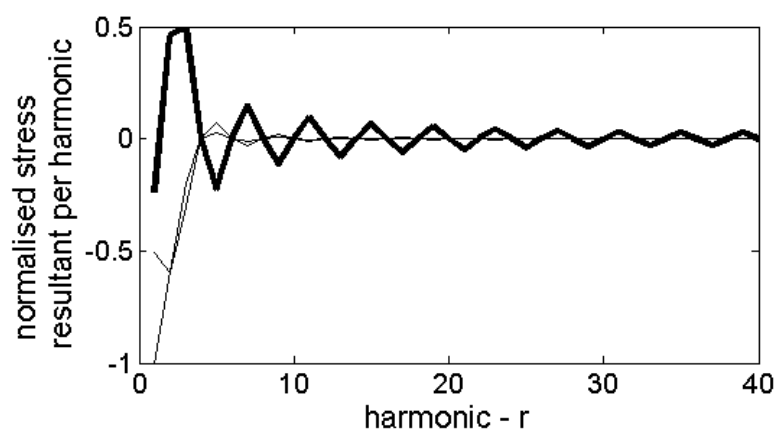

C1 Smooth

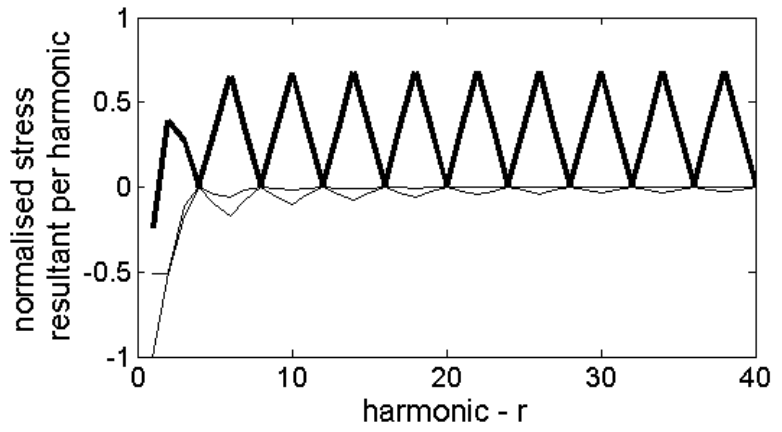

Triangular

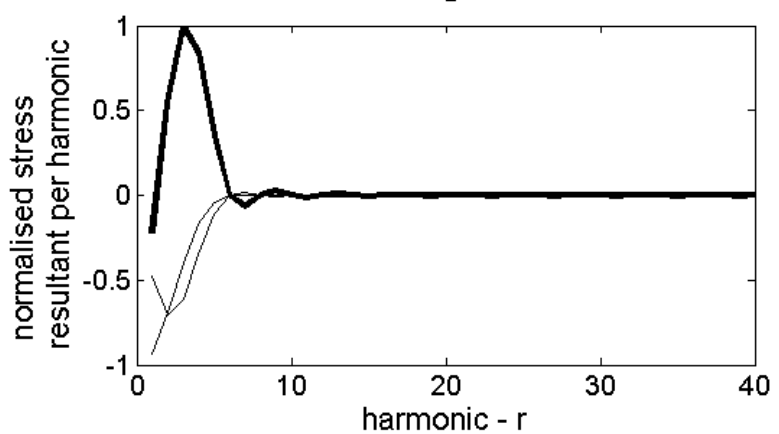

C2 Smooth

Fig. 6. Convergence of membrane stress resultants at higher harmonics - without sigma smoothing

The divergence of the meridional membrane stress resultant harmonics can be clearly seen in Fig. 6. The series diverges for the rectangular function and oscillates without converging for the triangular function, making these shapes unsuitable for use. This was expected, based on earlier observations concerning Eqs 9 to 11. The C1 smooth shape converges satisfactorily after approximately 50 harmonics, but more slowly than the $\mathrm{C} 2$ smooth depression which has converged after less than 20. Sigma smoothing generally helps to improve convergence (Fig. 7), most notably for the triangular function which now converges, though many terms are necessary to achieve this. The rectangular shape remains divergent and cannot be used. 
Published in: Thin-Walled Structures, 49(7), 902-912.

DOI: http://dx.doi.org/10.1016/j.tws.2011.02.016
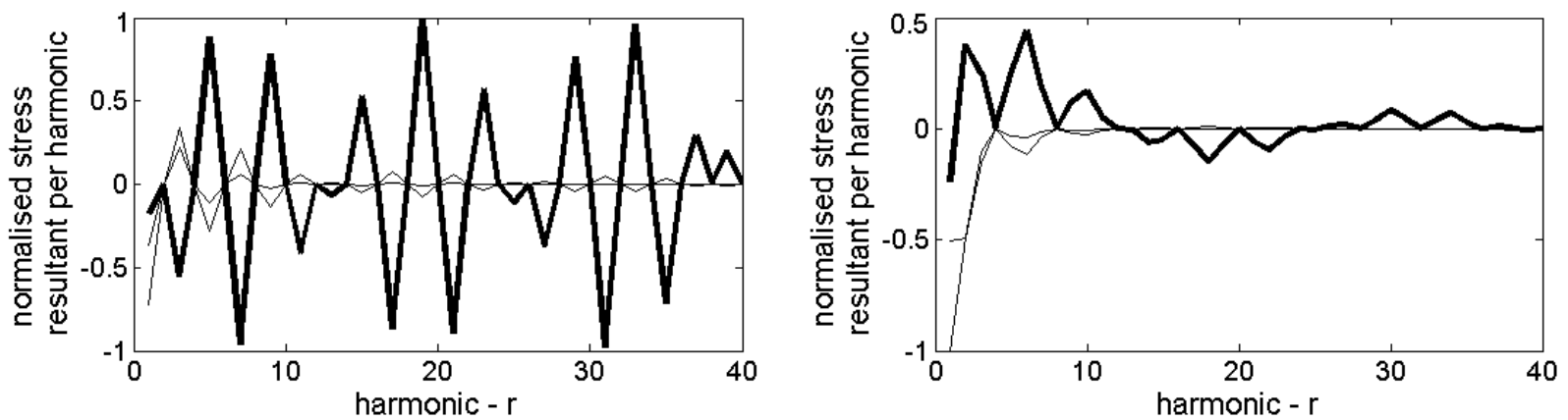

Rectangular

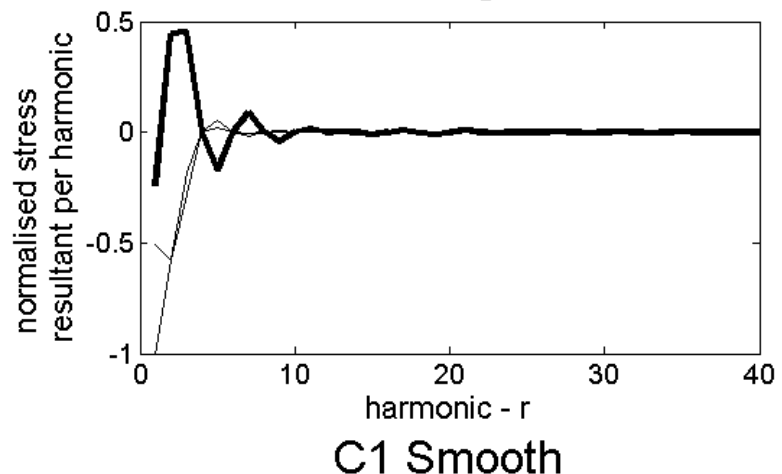

Triangular

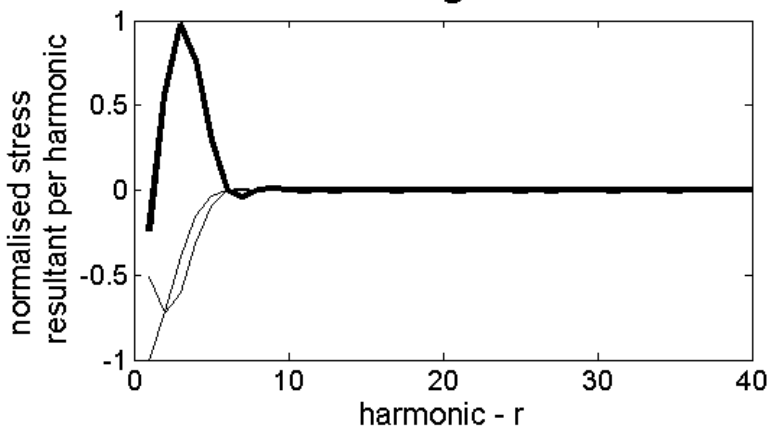

C2 Smooth

Fig. 7. Convergence of membrane stress resultants at higher harmonics - with sigma smoothing

The above comparison clearly demonstrates the importance of a careful choice of function for the circumferential pressure depression. From the above, only the $\mathrm{C} 1$ and $\mathrm{C} 2$ smooth functions are suitable, and these alone are used in the following finite element verifications.

\section{Verification against a finite element model}

The membrane stress resultants derived from membrane theory were next compared against a linear elastic bending analysis performed using the ABAQUS finite element package [1]. Half of the conical hopper with a plane of symmetry at $\theta=0^{\circ}$ was modelled with eight-node reduced-integration S8R5 elements. The transition radius to thickness ratio was 5000 making the hopper wall very thin to emphasise the membrane behaviour.

The results were made dimensionless by dividing by the maximum value of the meridional membrane stress resultant at the transition under axisymmetric loading, easily found by equilibrium of the forces due to the solid in the hopper and the cylinder. It is given by:

$N_{\phi r e f}=\left(p_{v f t}+\frac{\gamma L}{3}\right)\left(\frac{L}{2}\right) \sec \beta \tan \beta$

The full set of dimensionless stress resultants are then defined here as: 
Published in: Thin-Walled Structures, 49(7), 902-912.

DOI: http://dx.doi.org/10.1016/j.tws.2011.02.016

$$
N_{\theta \mathrm{dim}}=\frac{N_{\theta}}{N_{\phi r e f}} ; N_{\phi \theta \mathrm{dim}}=\frac{N_{\phi \theta}}{N_{\phi r e f}} ; N_{\phi \mathrm{dim}}=\frac{N_{\phi}}{N_{\phi r e f}}
$$

\subsection{Axisymmetric pressures}

Three hoppers of different steepness with cone apex half angles $\beta$ of $20^{\circ}$ (steep), $45^{\circ}$ (intermediate) and $60^{\circ}$ (shallow) were analysed to illustrate the relative influence of bending in steep and shallow hoppers. The bottom edge was kept free of all restraint, while the transition was simply-supported (restrained meridional, radial and circumferential displacements, free rotations).

The circumferential membrane stress resultants are shown in Fig. 8 and clearly follow the membrane theory predictions very closely. Close to the transition, however, these stresses are significantly affected by bending at the hopper top. It can be seen that bending effects penetrate slightly further into the shell as the apex half-angle increases (as predicted by Eq. 7), giving a larger zone of mismatch between the two calculations for shallow hoppers.

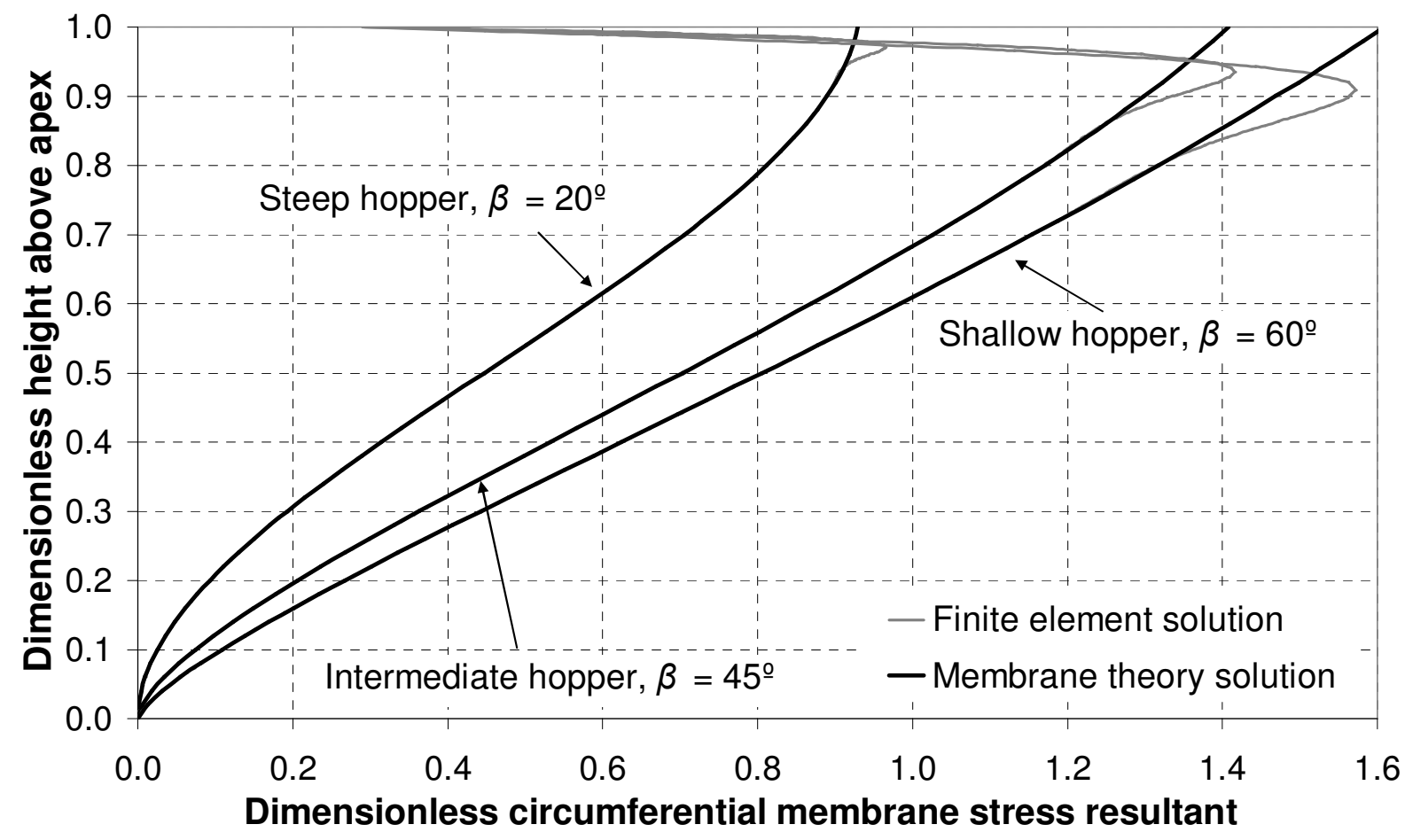

Fig. 8. Axial distribution of the circumferential membrane stress resultant under axisymmetric pressures

The meridional membrane stress resultants are shown in Fig. 9 and the correlation with the membrane theory predictions are remarkably close throughout the entire hopper. Failure in the 
Published in: Thin-Walled Structures, 49(7), 902-912.

DOI: http://dx.doi.org/10.1016/j.tws.2011.02.016

hopper under axisymmetric loading is commonly by tensile rupture at the transition, which is governed by the meridional membrane stress state. This can be seen to be completely governed by membrane action with negligible bending.

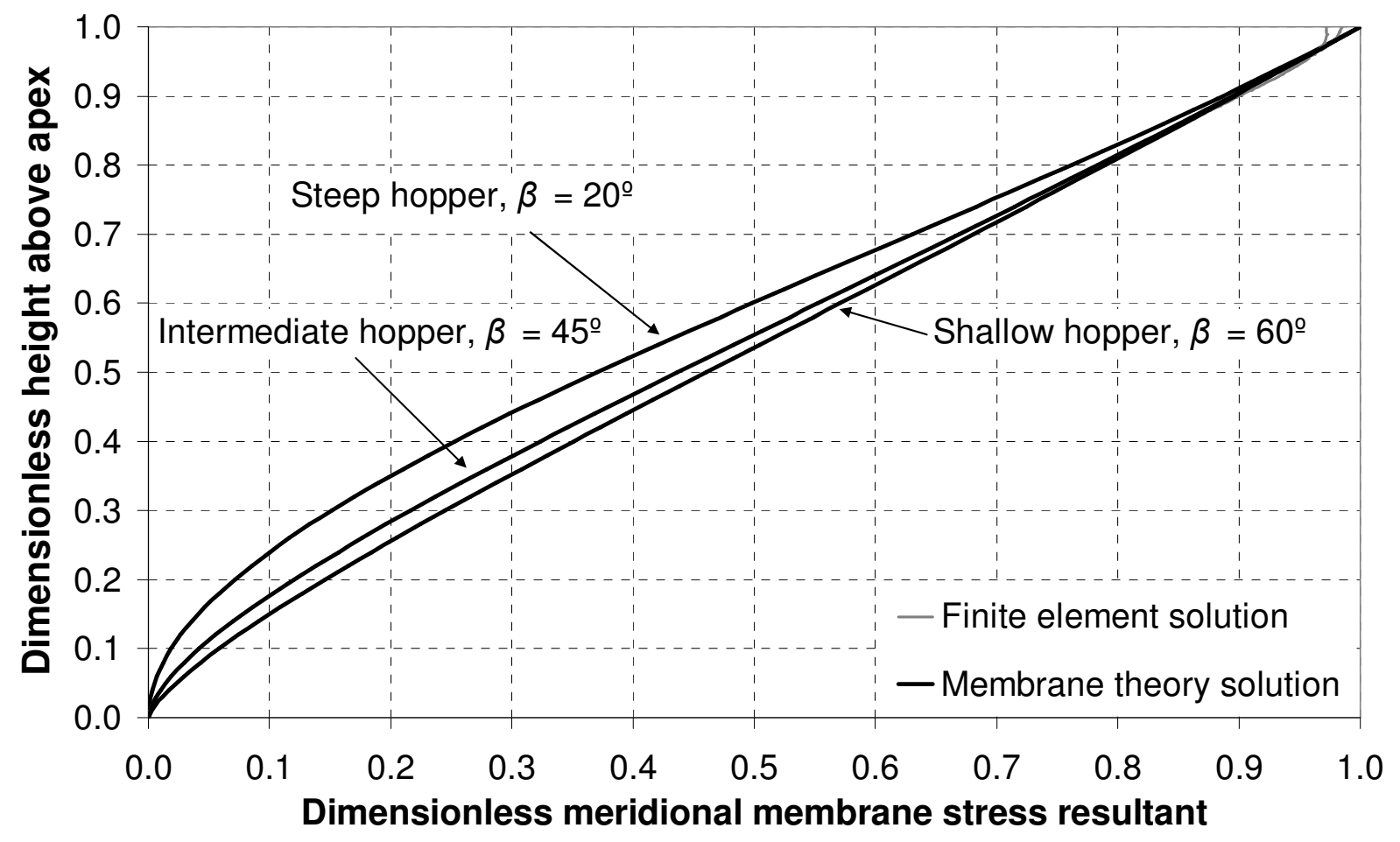

Fig. 9. Axial distribution of the meridional membrane stress resultant under axisymmetric pressures

\subsection{Non-symmetric pressures}

A steep hopper with an apex half-angle of $\beta=25^{\circ}$ was modelled with axisymmetric and unsymmetrical eccentric discharge pressures using the $\mathrm{C} 1$ and $\mathrm{C} 2$ smooth functions. A value of $\eta$ of -0.5 ( $\eta$ negative to give a reduction in pressures) and a fairly large value of $\theta_{0}=60^{\circ}$ (Fig. 1) were chosen here.

The lower half of the hopper is dominated by bending behaviour that is highly sensitive to the base boundary conditions, which are difficult to identify properly for a conical hopper. A base restraint against meridional displacements was assumed here, though it is free to rotate and displace circumferentially. In practice there would be a stiffening ring present at the outlet which would exhibit similar behaviour whilst not strictly restraining meridional movement, though this is difficult to implement properly as a numerical boundary condition. The upper half of the hopper is in an almost perfect membrane state, with stresses that are largely 
Published in: Thin-Walled Structures, 49(7), 902-912.

DOI: http://dx.doi.org/10.1016/j.tws.2011.02.016

independent of either boundary. The transition was once again assumed to be simplysupported.

The axial distributions of circumferential membrane stress resultants through the centre of the depression are shown in Fig. 10. Circumferential distributions of this stress resultant are shown in Fig. 11 at $0.75 \mathrm{~L}$ and $0.50 \mathrm{~L}$. The correlation between membrane theory and the computation is excellent at locations away from either boundary. The circumferential membrane stress resultants closely follow the shape of the pressure distribution as they depend linearly on the normal pressures (see Eqs 13 and 22). There are some minor bending effects, most notably at the edges of the $\mathrm{C} 2$ smooth depression at midheight (see Fig. 11). The C2 $\cos ^{4}$ function peaks more steeply at $\theta=0^{\circ}$ than the $\mathrm{C} 1 \cos$ function and has almost double the curvature (see Fig. 12) making it more susceptible to inducing bending that will disturb the membrane stress state even if the depression amplitude $\eta$ is the same.

The circumferential distribution of the membrane shear stress resultants just below the transition $(0.99 L)$ and at midheight are shown in Fig. 13. The shears achieve a maximum at the transition, with little interference from bending stresses, and the correlation between the present theory and the numerical calculation is very high. As these values have been found using the boundary condition of $N_{\phi \theta r}=0$ at the outlet, the distribution of the shears is evidently not sensitive to either boundary condition. The $\mathrm{C} 2$ function results in marginally higher values of shear than the $\mathrm{C} 1$ function due to its higher second derivative. 
Published in: Thin-Walled Structures, 49(7), 902-912.

DOI: http://dx.doi.org/10.1016/j.tws.2011.02.016

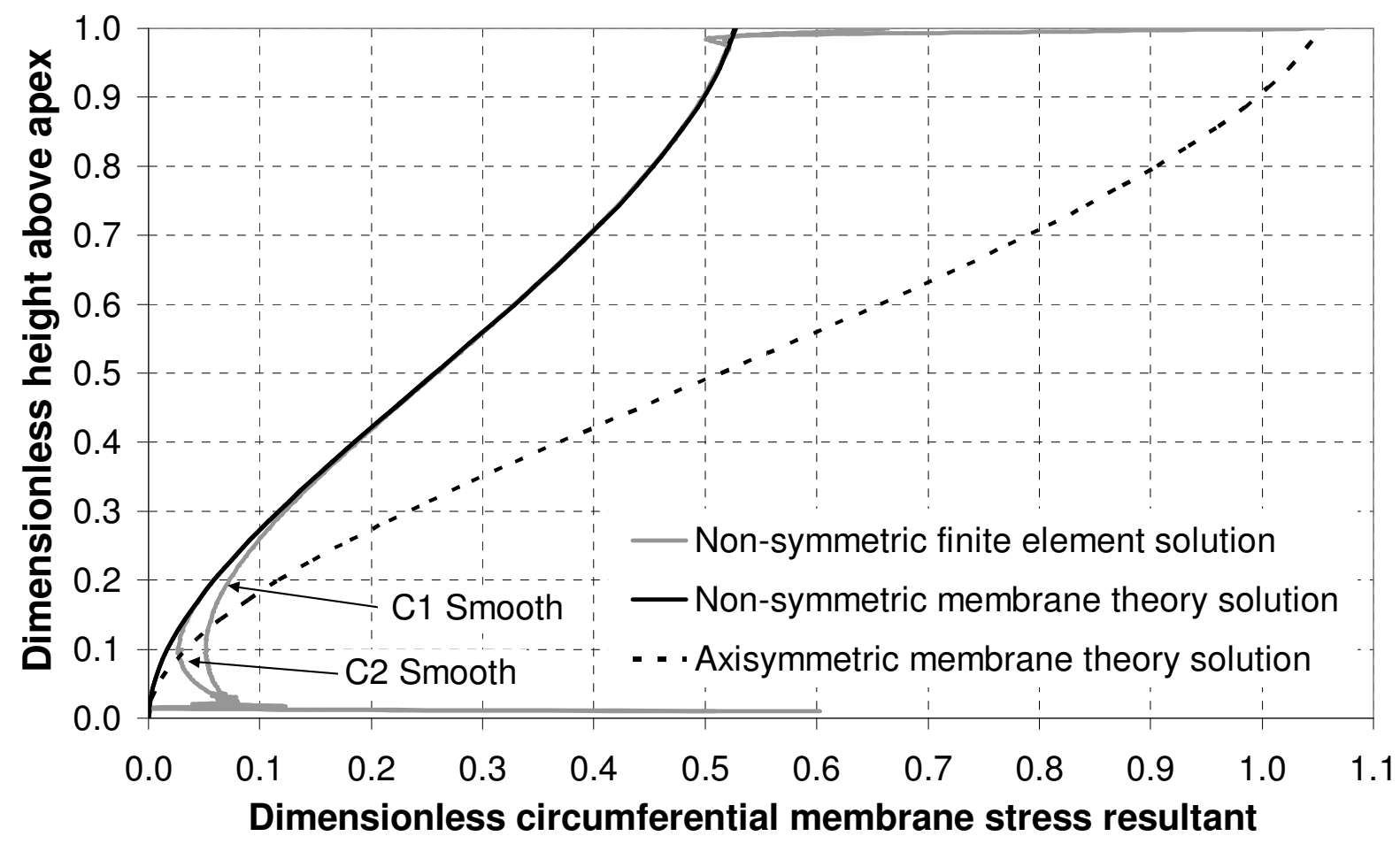

Fig. 10. Axial distribution of select circumferential membrane stress resultants through the centre of the depression

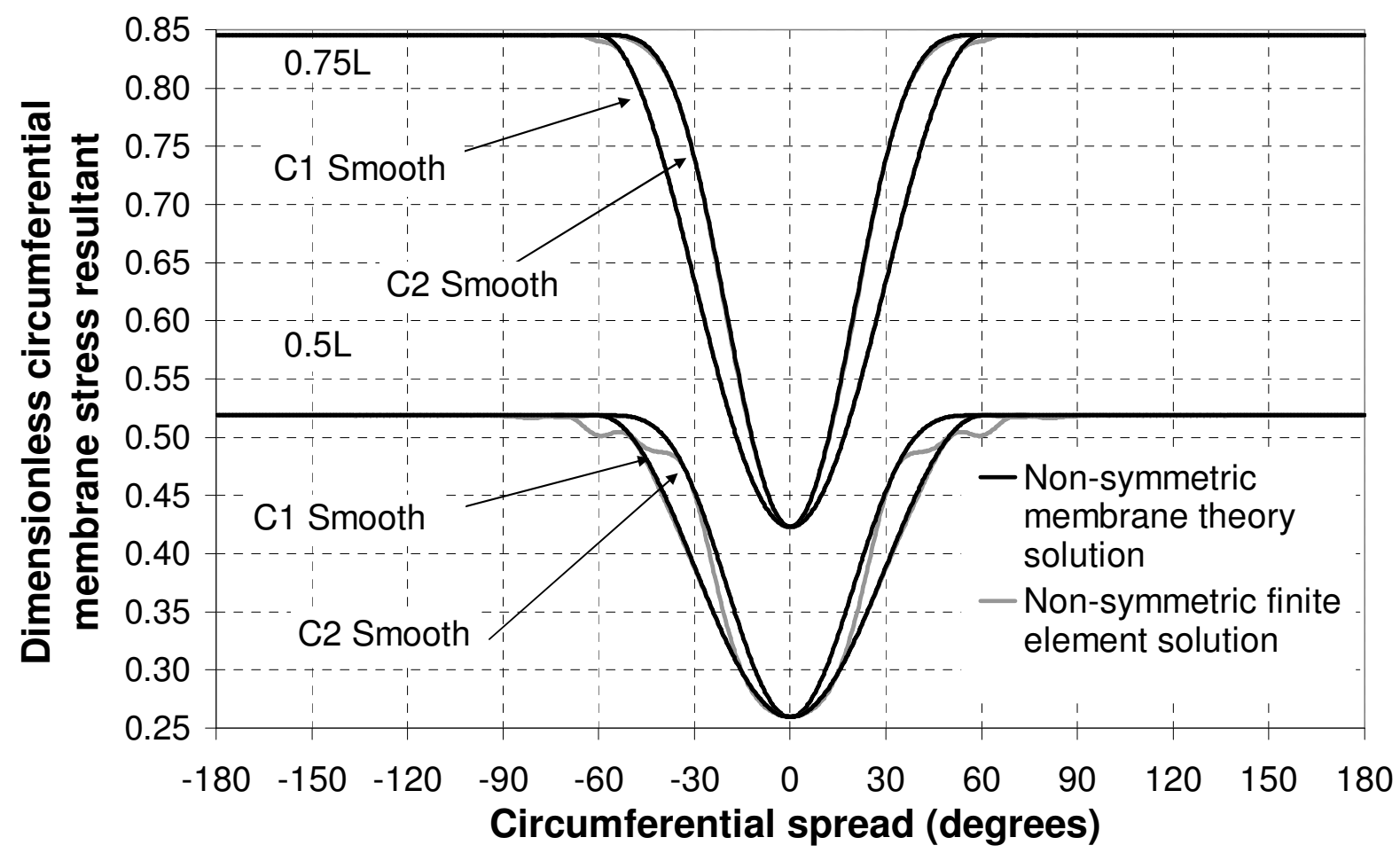

Fig. 11. Circumferential distribution of the circumferential membrane stress resultant due to the pressure depressions at two levels in the hopper 
Published in: Thin-Walled Structures, 49(7), 902-912.

DOI: http://dx.doi.org/10.1016/j.tws.2011.02.016

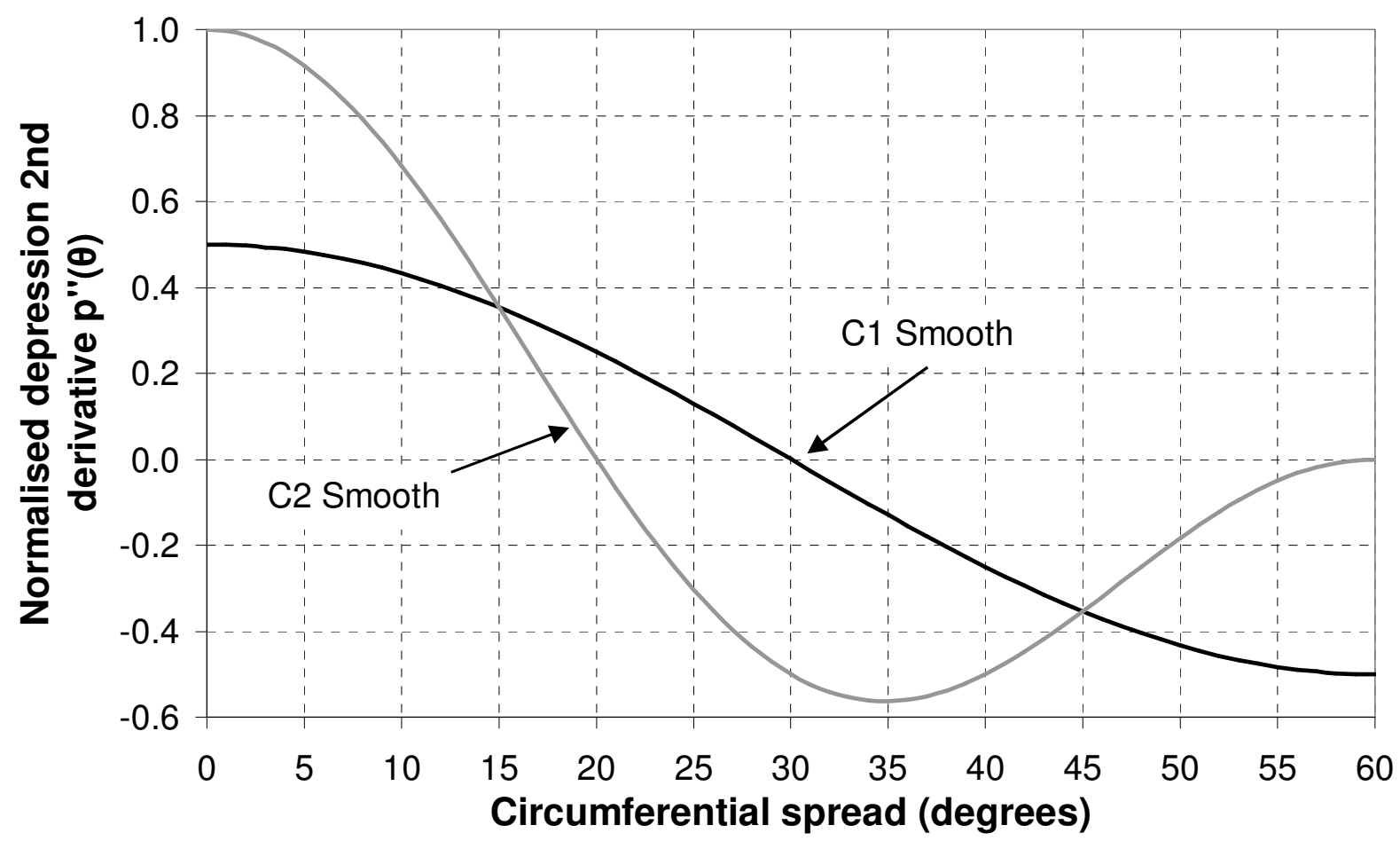

Fig. 12. Circumferential distribution of the second derivative of the depression function

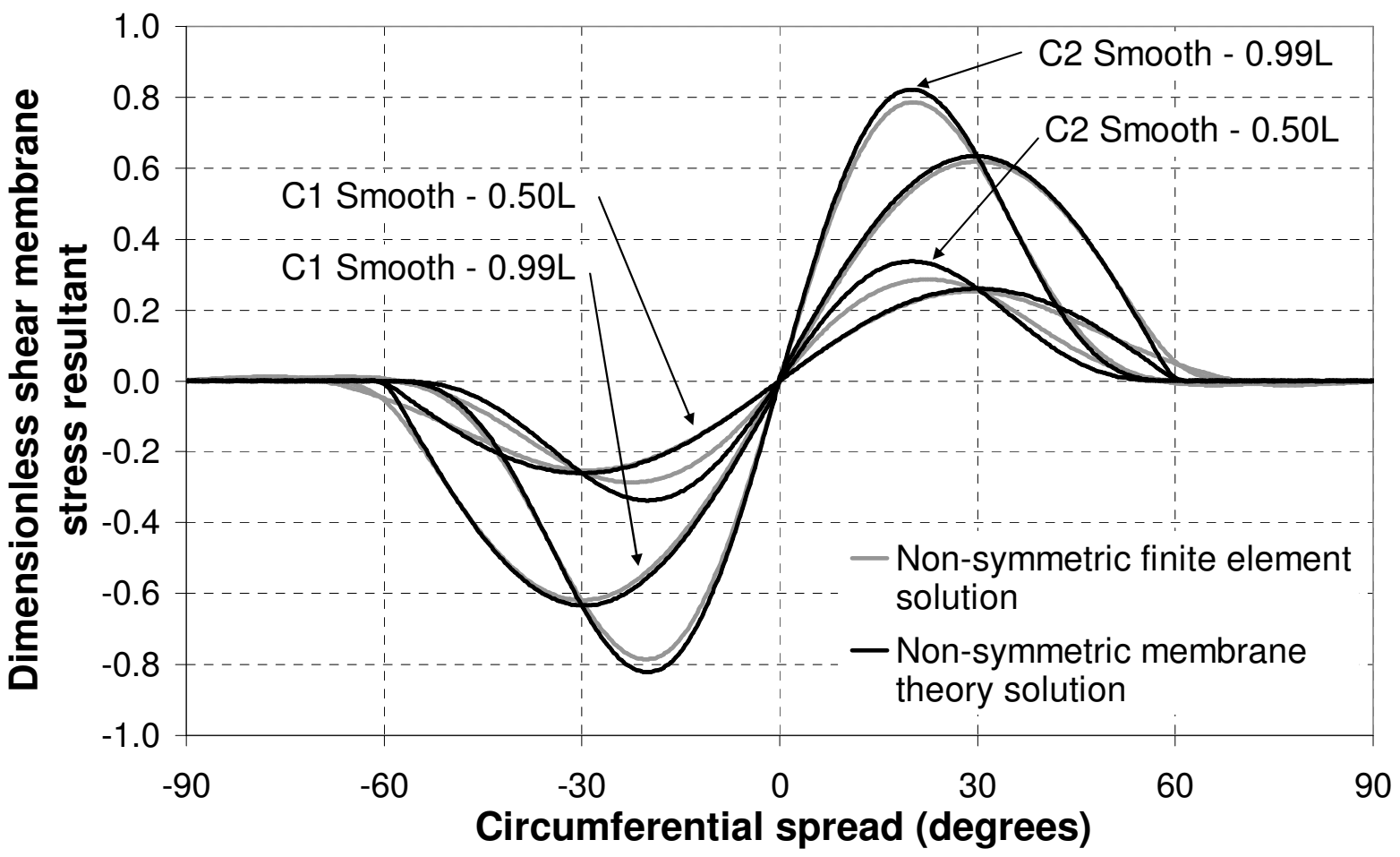

Fig. 13. Circumferential distribution of the membrane shear stress resultant at two levels in the hopper due to the pressure depressions 
Published in: Thin-Walled Structures, 49(7), 902-912.

DOI: http://dx.doi.org/10.1016/j.tws.2011.02.016

The axial distributions of the meridional membrane stress resultant through the centre of the depression, with and without the base boundary condition (Eq. 35) of restrained meridional displacement at the outlet, are shown in Figs 14 and 15 for the $\mathrm{C} 1$ and $\mathrm{C} 2$ smooth depressions respectively. The membrane theory result for non-symmetric pressures is obtained after double circumferential differentiation of the pressure distribution, where boundary conditions and convergence properties become important. So it is difficult to get a perfect match between membrane theory and the finite element calculation.

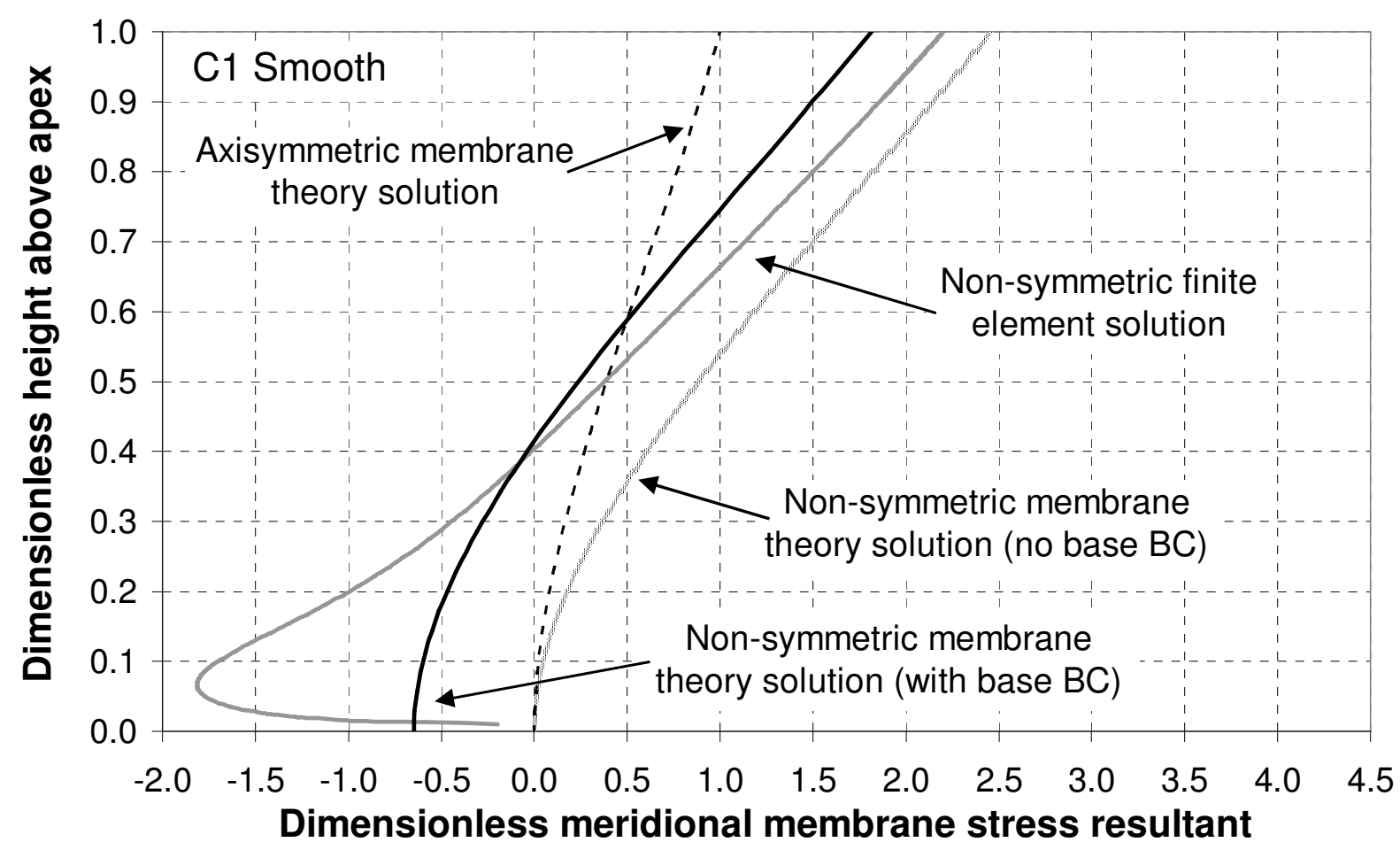

Fig. 14. Meridional membrane stress resultants through the centre of the C1 Smooth depression from a finite element analysis and membrane theory (with and without a base meridional displacement restriction) 
Published in: Thin-Walled Structures, 49(7), 902-912.

DOI: http://dx.doi.org/10.1016/j.tws.2011.02.016

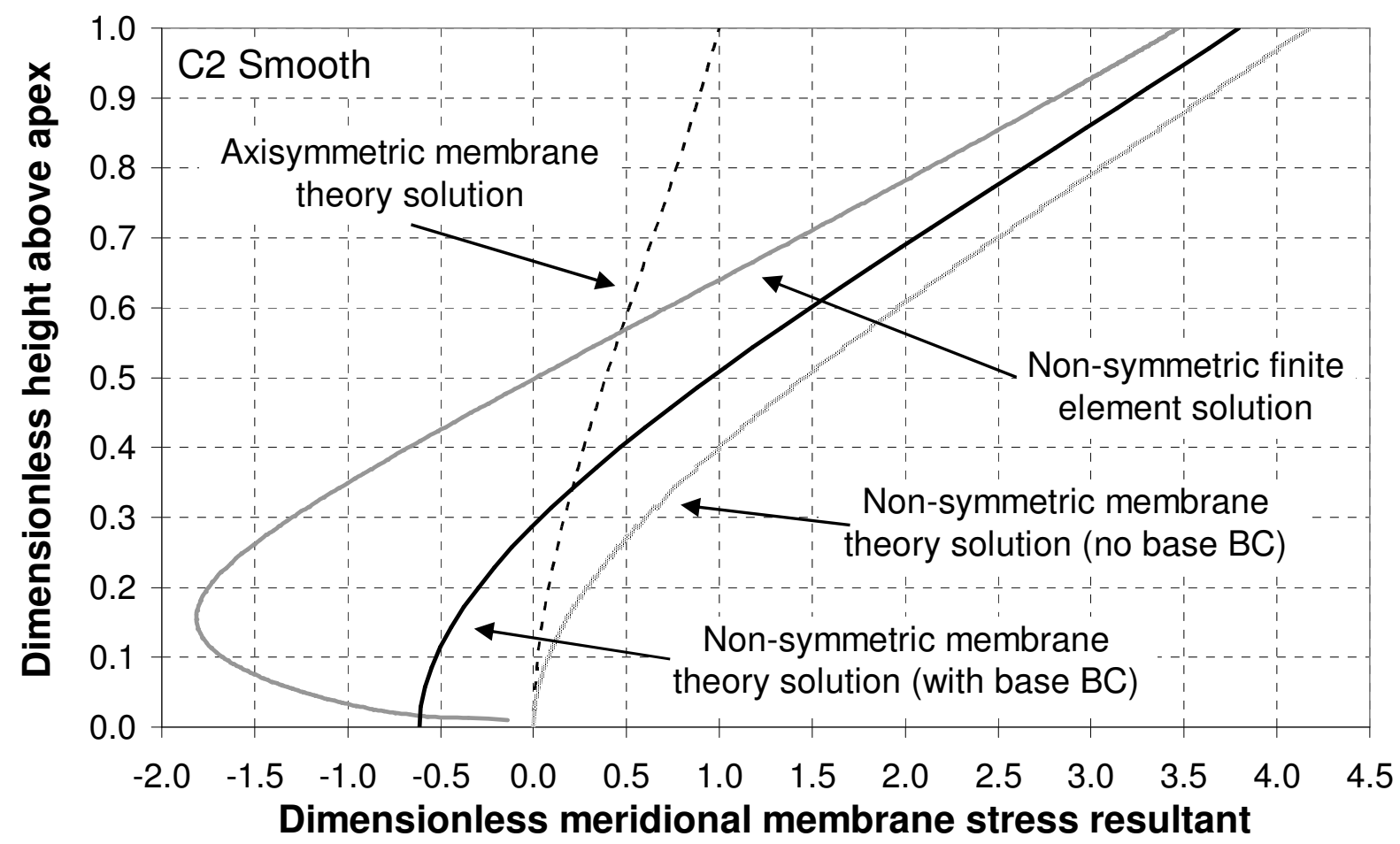

Fig. 15. Meridional membrane stress resultants through the centre of the C2 Smooth depression from a finite element analysis and membrane theory (with and without a base meridional displacement restriction)

According to this simple membrane theory model for eccentric discharge, the peak value of the meridional membrane stress at the transition is almost $100 \%$ larger than the axisymmetric loading value for the $\mathrm{C} 1$ smooth depression, and over $200 \%$ larger for the $\mathrm{C} 2$ smooth depression. The order of magnitude of these changes is confirmed by the finite element analysis. This rise in stresses drastically increases the risk of failure by rupture at the transition, which may lead to unzipping of the hopper at this location and complete loss of contents (Fig. 16). Additionally, compressive membrane stresses develop near the bottom of the hopper, as well as to the sides of the depression near the transition (Fig. 17), introducing the possibility of buckle formation. The shape of the circumferential distribution is seen to invert between the form near the transition and that at midheight (Fig. 18), though the membrane solution for the steeper $\mathrm{C} 2$ depression has difficulty in capturing this change. 
Published in: Thin-Walled Structures, 49(7), 902-912.

DOI: http://dx.doi.org/10.1016/j.tws.2011.02.016

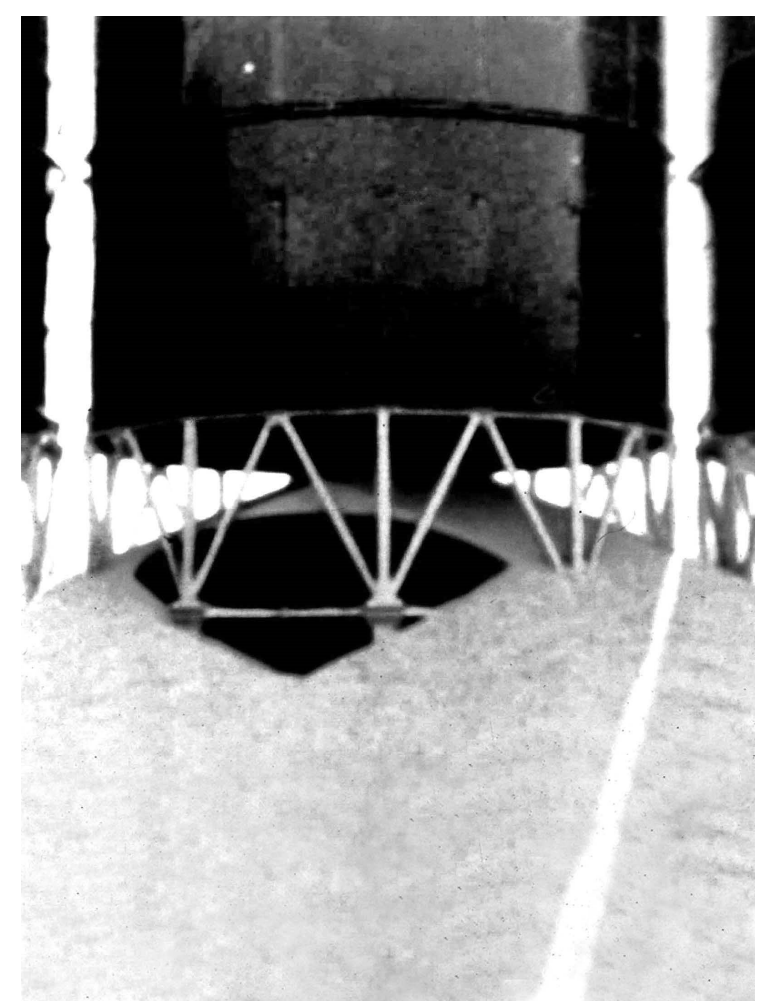

Fig. 16. Rupture failure of a hopper, leading to unzipping of the transition and complete loss of contents (photo courtesy of J.M. Rotter)

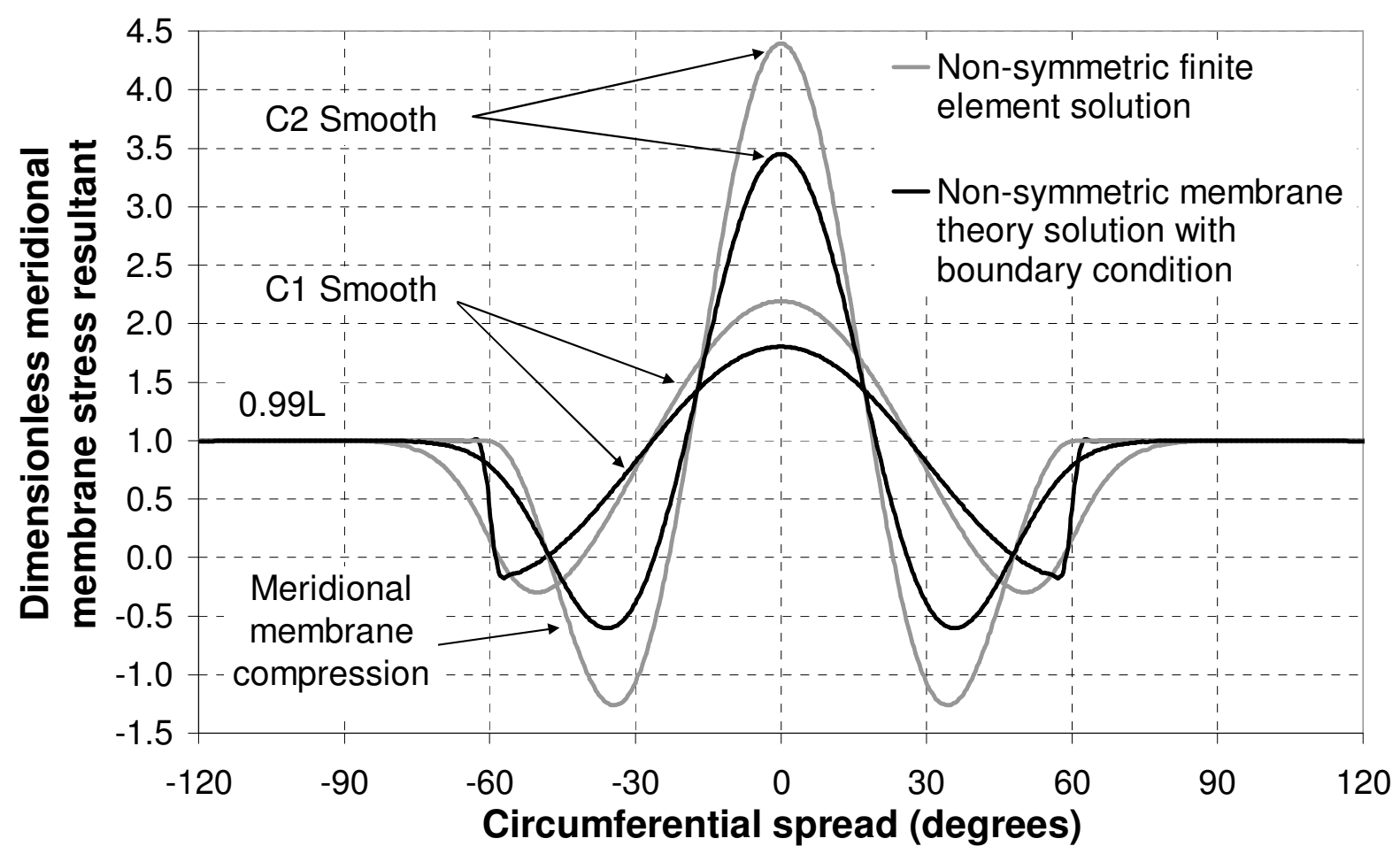

Fig. 17. Circumferential distribution of the meridional membrane stress resultant for the pressure depressions just below the transition 
Published in: Thin-Walled Structures, 49(7), 902-912.

DOI: http://dx.doi.org/10.1016/j.tws.2011.02.016

The compressive region to the sides of the depression near the transition is very small, highly dependent on the value of $\eta$ and is unlikely to develop for smaller channels or more realistic, thicker walls. Whilst there is the possibility of buckles forming here, they are likely to be small and consequently only forming under high stresses. It is therefore a less likely consequence than rupture at the transition.

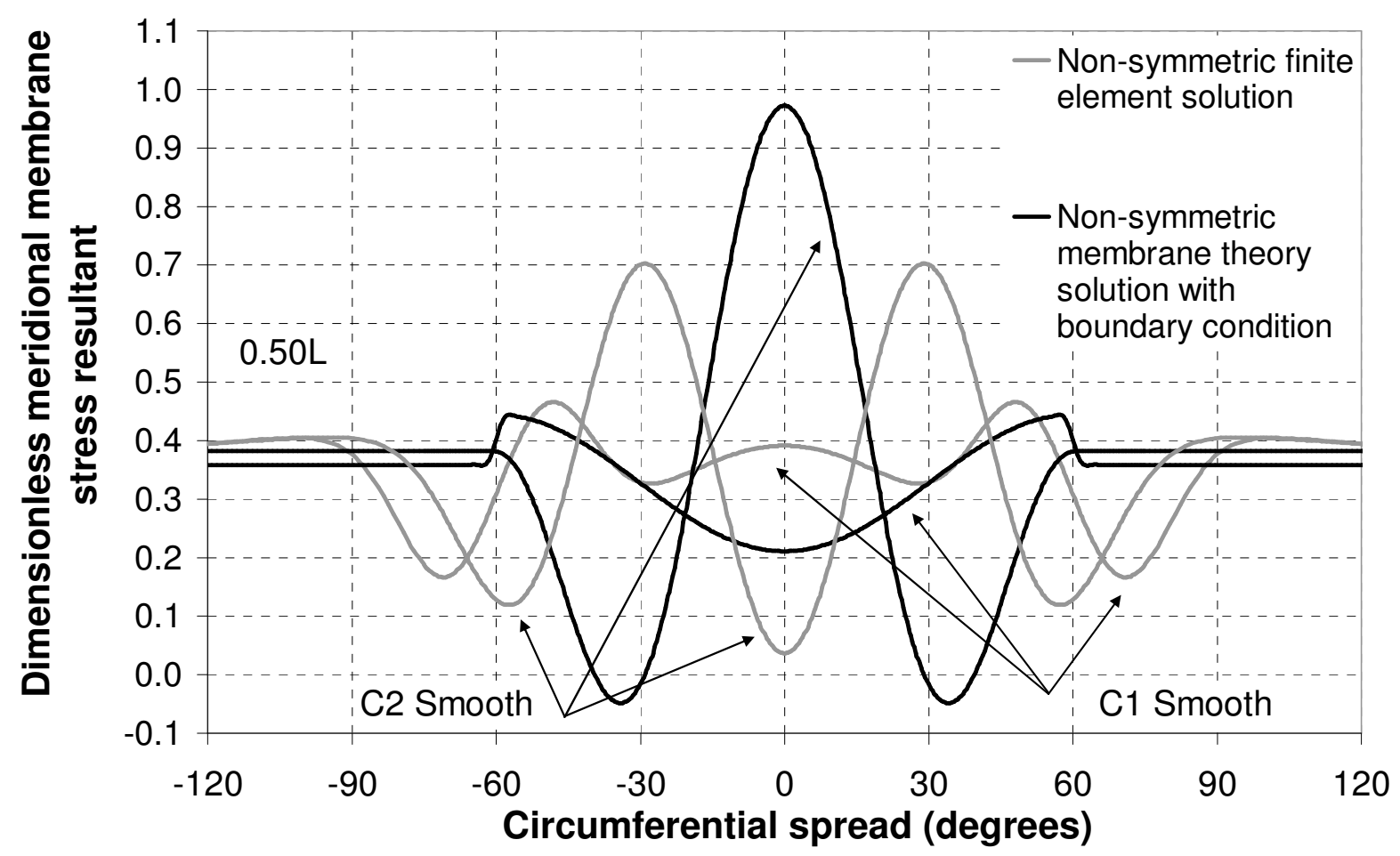

Fig. 18. Circumferential distribution of the meridional membrane stress resultant for the pressure depressions at midheight

The higher value of the second derivative in the $\mathrm{C} 2$ smooth function (see Fig. 12) has little effect on the magnitudes of the circumferential and shear membrane stresses, but its effect on the meridional membrane stresses is surprisingly large. The peak magnitudes of $N_{\phi}$ at the transition with the steeper C2 function (Fig. 15) are consistently double what they would be with the $\mathrm{C} 1$ function (Fig. 14). These magnitudes are all linearly dependent on the depression amplitude $\eta$ (Fig. 19) and increase exponentially with a decreasing apex half-angle $\beta$ (Fig. 20). The design of steeper hoppers should therefore have a special provision for the possibility of eccentric flow in design, as these are most susceptible to major damage if this occurs.

The membrane theory solution, with the boundary condition of restrained meridional displacements at the outlet (Eq. 35), is thus able to capture the dominant structural behaviour 
Published in: Thin-Walled Structures, 49(7), 902-912.

DOI: http://dx.doi.org/10.1016/j.tws.2011.02.016

qualitatively, with a moderately close match to the numerical calculation. The discrepancies are partly due to the uncertainty of the boundary condition at the outlet and partly due to bending effects. The second derivative discontinuity at each edge of the $\mathrm{C} 1$ smooth depression is clearly visible in Figs 17 and 18, while the C2 smooth depression has no such problem.

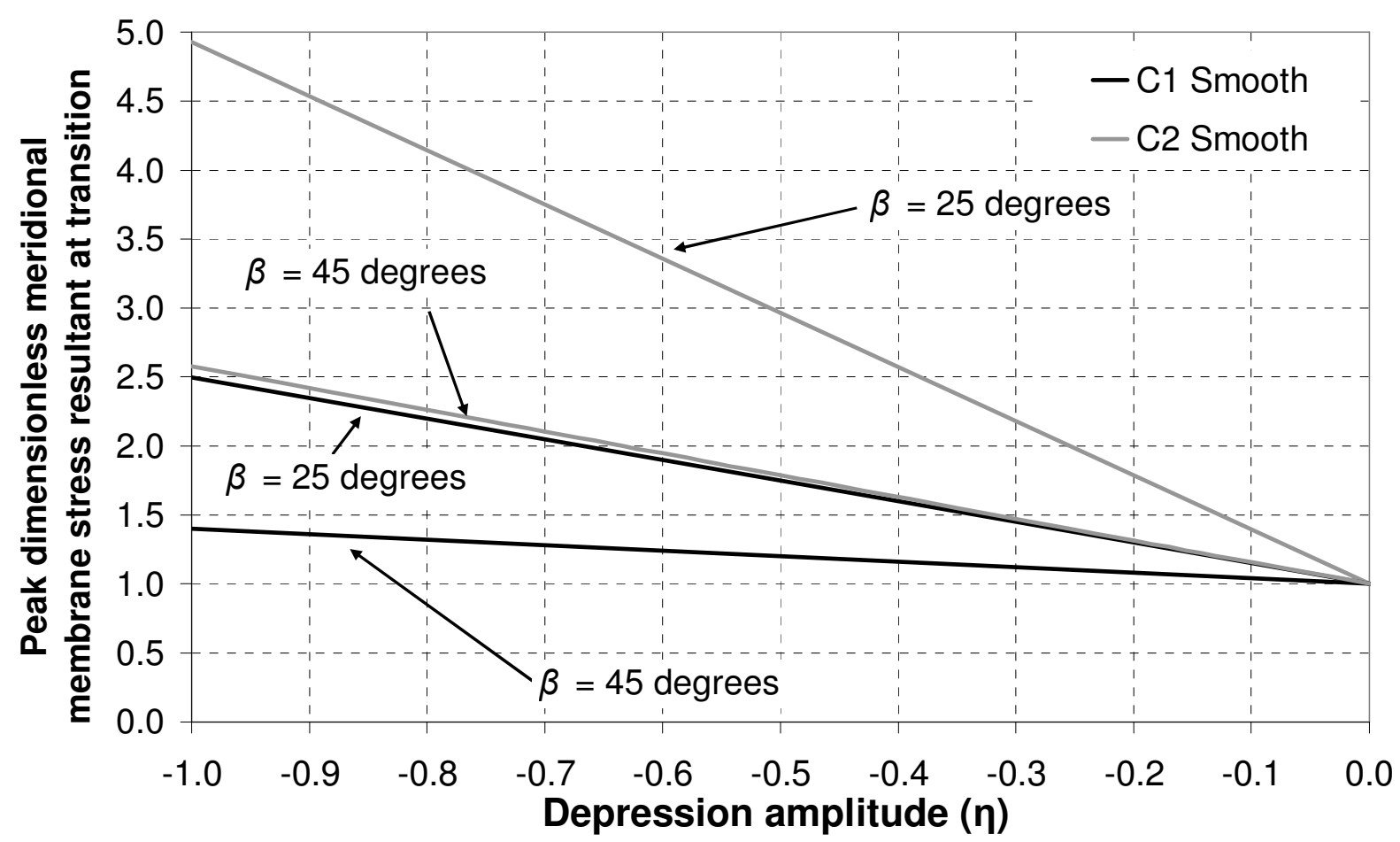

Fig. 19. Variation with the depression amplitude of the peak dimensionless meridional membrane stress resultant calculated with membrane theory at the transition at the centre of the channel 
Published in: Thin-Walled Structures, 49(7), 902-912.

DOI: http://dx.doi.org/10.1016/j.tws.2011.02.016

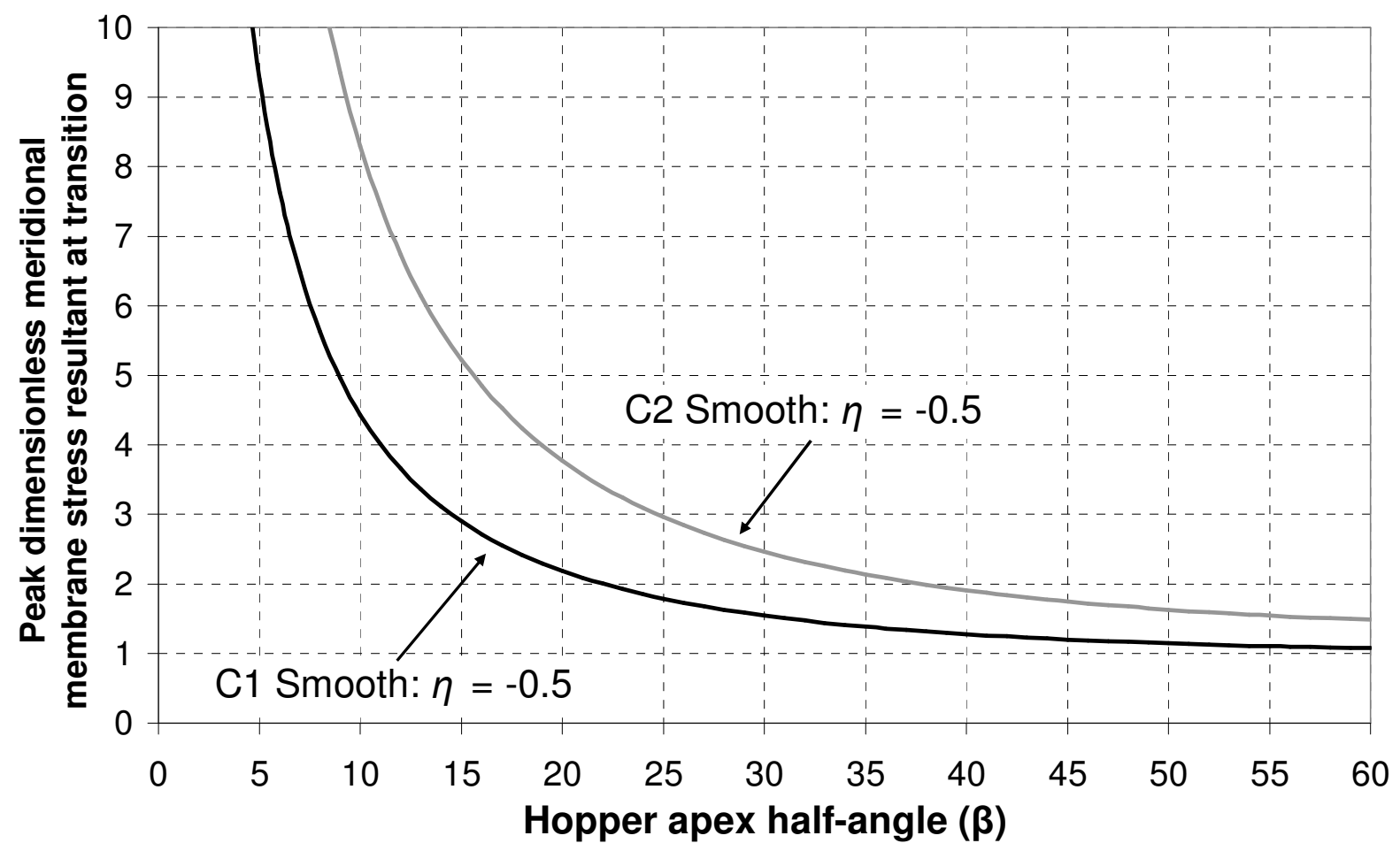

Fig. 20. Variation with the hopper apex half-angle of the peak dimensionless meridional membrane stress resultant calculated with membrane theory at the transition at the centre of the channel

The choice of an appropriate depression shape is currently open, as there is no known experimental data that can give an indication of a realistic form. As both the $\cos$ and $\cos ^{4}$ functions could be fitted with similar certainty to any approximately bell-shaped set of experimental pressure data, much detail is needed in such a test. Yet the two forms result in very different stress states, with one predicting hopper rupture much earlier than the other. It is clear that good hopper design to avoid rupture under eccentric discharge flows is in need of very careful and detailed experimental data to determine an appropriate form.

\section{Conclusions}

This paper has presented an initial study of the structural consequences of an accidental eccentric flow channel of granular solids in a right conical hopper using shell membrane theory verified against a finite element analysis. The pressures due to the flow channel have been characterised as a reduction in normal pressures and frictional tractions varying as a smooth local $\cos$ or $\cos ^{4}$ function. 
Published in: Thin-Walled Structures, 49(7), 902-912.

DOI: http://dx.doi.org/10.1016/j.tws.2011.02.016

The hopper is subject to biaxial tension under symmetrical loading, and failure is normally by tensile material rupture at the hopper transition junction, governed by meridional membrane stresses. The strength is limited by the ultimate tensile stress of the shell wall material. It has been found in this study that eccentric discharge dramatically increases the peak meridional membrane stress at the centre of the flow channel at the transition, leading to a much higher risk of rupture. Compressive membrane stresses may develop at the edges of the depression and in the bottom half of the silo, introducing a possibility of buckling, but the region of compressive stresses is very small so only a small buckle can form, with a consequent requirement for a high stress to induce it.

The peak meridional membrane stress at the top of the hopper is greatly increased by eccentric discharge. Since failure of the joint at this location is by rupture, which is generally not a ductile failure mode, it is likely that a local failure would lead to progressive rupture of the whole joint (unzipping) with complete loss of the hopper. The peak stress at this point varies linearly with the depression amplitude and inversely exponentially with the hopper apex halfangle, becoming very high indeed for steep hoppers. The design of hoppers should therefore be extended to accommodate the possibility of accidental eccentric discharge, due to the severity of the structural consequences.

The membrane theory equations capture the structural behaviour of the hopper reasonably well and give a satisfactory correlation with results from a finite element analysis. It should therefore be possible to develop some simple design advice for this condition, based on the solutions presented in this paper.

The two seemingly similar versions of the shape of the pressure drop due to eccentric discharge give very different predictions of hopper rupture. It is therefore clear that very detailed experiments are needed to determine the precise shape of such a depression, so that its implications for structural design can be included in design with confidence. 
Published in: Thin-Walled Structures, 49(7), 902-912.

DOI: http://dx.doi.org/10.1016/j.tws.2011.02.016

\section{References}

[1] ABAQUS (2009). “ABAQUS V. 6.9.” Dassault Systèmes Simulia Corp., Providence, RI, USA.

[2] Bosley J., Scholfield C. \& Shook C.A. (1969). “An Experimental Study of Granular Discharge from Model Hoppers". Transaction of the Inst. Of Chem. Engineers, Vol. 47, T147T153.

[3] Budiansky B. \& Sanders J.L. (1963). "On the 'Best' First-Order Linear Shell Theory." Progress in Applied Mechanics, The Prager Anniversary Volume, Macmillan, 129-140.

[4] Carson J.W., Goodwill D.J. \& Bengston K.E. (1991). "Predicting the shape of flow channels in funnel flow bins and silos". Proceedings of the ACI Convention, Boston, Massachussetts, March, 12pp.

[5] Chen, J.F., Rotter, J.M., Ooi, J.Y. \& Zhong, Z. (2007) "Correlation between the flow pattern and wall pressures in a full scale silo", Engineering Structures, Vol. 29, pp 2308-2320.

[6] EN 1991-4 (2007). "Eurocode 1: Actions on Structures, Part 4: Silos and Tanks.” European Committee for Normalisation, Brussels.

[7] Enstad G. (1975). "On the Theory of Arching in Mass-Flow Hoppers". Chem. Eng. Sci., Vol. 30, N. 10, 1273-1283.

[8] Flügge W. (1957). "Statik und Dynamik der Schalen - zweite Auflage." Springer-Verlag, Berlin, Göttingen \& Heidelberg.

[9] Gould P.L. (1977). "Static analysis of shells." Lexington Books, D.C. Heath \& Co., Lexington.

[10] Jenike A.W. (1967). "Denting of Circular Bins with Eccentric Drawpoints". Jrnl. of the Struct. Div., ASCE, Vol. 93, No. ST1, 27-35. 
Published in: Thin-Walled Structures, 49(7), 902-912.

DOI: http://dx.doi.org/10.1016/j.tws.2011.02.016

[11] Jenkyn R.T. \& Goodwill D.J. (1987). “Silo Failure: Lessons to be Learned.” Engineering Digest, September, 17-22.

[12] Lanczos C. (1961). "Linear Differential Operators." D. Van Nostran Company Ltd., London, UK.

[13] Roberts A.W. \& Ooms M. (1983). "Wall Loads in Large Steel and Concrete Bins and Silos due to Eccentric Draw-Down and other Factors". Proc. $2^{\text {nd }}$ Int. Conf. on Design of Silos for Strength and Flow, 151-170.

[14] Rotter J.M. (1986). "The analysis of steel bins subject to eccentric discharge.” Proc. of the $2^{\text {nd }}$ Int. Conf. on Bulk Materials Storage, Handling and Transportation, IEAustralia, Wollongong, 264-271.

[15] Rotter J.M. (1987a). "Bending Theory of Shells for Bins and Silos." Trans. of Mech. Eng., IE Aust, ME12 (3), 147-159.

[16] Rotter J.M. (1987b). "Membrane Theory of Shells for Bins and Silos." Trans. of Mech. Eng., IE Aust, ME12 (3), 135-147.

[17] Rotter J.M. (2001a). "Guide for the Economic Design of Circular Metal Silos.", Spon Press, London \& New York.

[18] Rotter J.M. (2001b). "Pressures, stresses and buckling in metal silos containing eccentrically discharging solids." Festschrift Richard Greiner, celebration volume for the $60^{\text {th }}$ birthday of Prof. Richard Greiner, Institute for Steel and Shell Structures, TU Graz, Austria, 85-104.

[19] Sadowski A.J. (2010). "Modelling of Failures in Thin-Walled Metal Silos under Eccentric Discharge." PhD Thesis, The University of Edinburgh.

[20] Sadowski A.J. \& Rotter J.M. (2010). ”Study of Buckling in Steel Silos under Eccentric Discharge Flows of Stored Solids.” J. of Eng. Mech., ASCE, 136(6), 769-776. 
Published in: Thin-Walled Structures, 49(7), 902-912.

DOI: http://dx.doi.org/10.1016/j.tws.2011.02.016

[21] Safarian S. S. \& Harris E. C. (1990). "Empirical Method for Computing Bending Moments in Circular Silo Walls due to Asymmetric Flow." FIP/ISO paper N. 3/90, FIP/ISO TC98/SC3/WG5 on Loads due to Bulk Materials, Karlsruhe, pp 7.

[22] Seide P. (1975). "Small elastic deformations of thin shells." Noordhoff International, Leyden.

[23] Timoshenko S. \& Woinowsky-Kreiger S. (1959). "Theory of Plates and Shells.” McGrawHill Book Company, New York.

[24] Vidal P., Couto A., Aygua F. And Guaita M. (2006). "Influence of hopper eccentricity on discharge of cylindrical mass flow silos with rigid walls." J. of Eng, Mech., ASCE, 132(9), 1026-1033.

[25] Walker D.M. (1966). “An Approximate Theory for Pressure and Arching in Hoppers." Chem. Eng. Science, Vol. 28, 975-997.

[26] Wood J.G.M. (1983). "The Analysis of Silo Structures subject to Eccentric Discharge." Proc. $2^{\text {nd }}$ Internat. Conf. on Design of Silos for Strength and Flow, Stratford-upon-Avon, 132144. 
Published in: Thin-Walled Structures, 49(7), 902-912.

DOI: http://dx.doi.org/10.1016/j.tws.2011.02.016

\section{Appendix A - Notation}

\begin{tabular}{|c|c|c|}
\hline Symbol & Unit & Description \\
\hline$F$ & - & Hopper wall pressure ratio \\
\hline$f_{0}$ & - & Fourier series coefficient for the 0 -th harmonic $(r=0)$ \\
\hline$f_{\mathrm{r}}$ & - & Fourier series coefficient for the $r$-th harmonic $(r>0)$ \\
\hline$L$ & $\mathrm{~m}$ & Vertical height of hopper along $z$-axis \\
\hline$N_{\theta}$ & $\mathrm{Nm}^{-1}$ & Circumferential membrane stress resultant \\
\hline$N_{\phi \theta}$ & $\mathrm{Nm}^{-1}$ & Shear membrane stress resultant \\
\hline$N_{\phi}$ & $\mathrm{Nm}^{-1}$ & Meridional membrane stress resultant \\
\hline$N_{\theta 0, \phi \theta 0, \phi 0}$ & $\mathrm{Nm}^{-1}$ & 0-th harmonic of the relevant membrane stress resultant \\
\hline$N_{\theta \mathrm{r}, \phi \theta r, \phi r}$ & $\mathrm{Nm}^{-1}$ & $r$-th harmonic of the relevant membrane stress resultant \\
\hline$N_{\phi \theta \mathrm{r} 0, \phi \mathrm{r} 0}$ & $\mathrm{Nm}^{-1}$ & constants of integration, per harmonic \\
\hline$p_{\text {axi }}$ & $\mathrm{Nm}^{-2}$ & Axisymmetric component of normal pressures \\
\hline$p_{\max }$ & $\mathrm{Nm}^{-2}$ & Generic maximum pressure value \\
\hline$p_{\mathrm{n}}$ & $\mathrm{Nm}^{-2}$ & Distributed surface pressure normal to the hopper wall \\
\hline$p_{\mathrm{nr}}$ & $\mathrm{Nm}^{-2}$ & $r$-th harmonic of unsymmetrical normal pressures \\
\hline$p_{\mathrm{p}}$ & $\mathrm{Nm}^{-2}$ & Maximum magnitude of pressure depression at any height \\
\hline$p_{\mathrm{v}}$ & $\mathrm{Nm}^{-2}$ & Mean vertical stress in the solid inside the hopper \\
\hline$p_{\mathrm{vft}}$ & $\mathrm{Nm}^{-2}$ & Mean vertical stress in the solid at the transition, $z=L$ \\
\hline$p_{\theta}$ & $\mathrm{Nm}^{-2}$ & Distributed frictional traction in the circumferential direction \\
\hline$p_{\theta \mathrm{r}}$ & $\mathrm{Nm}^{-2}$ & $r$-th harmonic of circumferential pressures \\
\hline$p_{\phi}$ & $\mathrm{Nm}^{-2}$ & Distributed frictional traction in the meridional direction \\
\hline$p_{\phi r}$ & $\mathrm{Nm}^{-2}$ & $r$-th harmonic of meridional pressures \\
\hline$r$ & - & Fourier harmonic number \\
\hline$R$ & $\mathrm{~m}$ & Silo radius, hopper radius at transition \\
\hline$t$ & $\mathrm{~m}$ & Hopper wall thickness \\
\hline$z$ & $\mathrm{~m}$ & Vertical coordinate \\
\hline$\beta$ & angle & Hopper apex half-angle \\
\hline$\gamma$ & $\mathrm{Nm}^{-3}$ & Bulk unit weight of stored solid \\
\hline$\eta$ & - & $\begin{array}{l}\text { Fraction of local normal pressures as a measure of amplitude of } \\
\text { the pressure depression }\end{array}$ \\
\hline$\theta$ & angle & Circumferential coordinate \\
\hline$\theta_{0}$ & angle & Circumferential extent of applied depression from centre of the \\
\hline
\end{tabular}


Published in: Thin-Walled Structures, 49(7), 902-912.

DOI: http://dx.doi.org/10.1016/j.tws.2011.02.016

$\begin{array}{lll}\lambda & \mathrm{m} & \text { Meridional bending half-wavelength } \\ \mu & - & \text { Wall friction coefficient } \\ v & - & \begin{array}{l}\text { Poisson's ratio of hopper wall material } \\ \sigma_{\theta}\end{array} \\ \sigma_{\phi} & \mathrm{Nm}^{-2} & \text { Circumferential membrane stress } \\ \tau_{\phi \theta} & \mathrm{Nm}^{-2} & \text { Meridional membrane stress } \\ \phi & \mathrm{Nm}^{-2} & \text { Shear membrane stress } \\ & - & \text { Meridional coordinate and slope of cone wall }\end{array}$

